\title{
Hippo/YAP signaling pathway protects against neomycin-induced hair cell damage in the mouse cochlea
}

\author{
Maohua Wang ${ }^{1,2,6}$ (1) $\cdot$ Ying Dong ${ }^{2} \cdot$ Song Gao ${ }^{3} \cdot$ Zhenhua Zhong $^{4} \cdot$ Cheng Cheng $^{3} \cdot$ Ruiying Qiang $^{2} \cdot$ Yuhua Zhang $^{2}$.

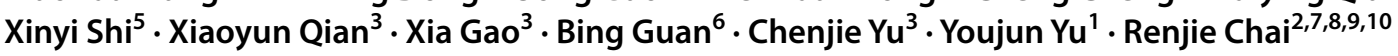

Received: 24 August 2021 / Revised: 23 October 2021 / Accepted: 8 November 2021 / Published online: 19 January 2022

(c) The Author(s) 2022

\begin{abstract}
The Hippo/Yes-associated protein (YAP) signaling pathway has been shown to be able to maintain organ size and homeostasis by regulating cell proliferation, differentiation, and apoptosis. The abuse of aminoglycosides is one of the main causes of sensorineural hearing loss (SSNHL). However, the role of the Hippo/YAP signaling pathway in cochlear hair cell (HC) damage protection in the auditory field is still unclear. In this study, we used the YAP agonist XMU-MP-1 (XMU) and the inhibitor Verteporfin (VP) to regulate the Hippo/YAP signaling pathway in vitro. We showed that YAP overexpression reduced neomycin-induced HC loss, while downregulated YAP expression increased HC vulnerability after neomycin exposure in vitro. We next found that activation of YAP expression inhibited C-Abl-mediated cell apoptosis, which led to reduced $\mathrm{HC}$ loss. Many previous studies have reported that the level of reactive oxygen species (ROS) is significantly increased in cochlear HCs after neomycin exposure. In our study, we also found that YAP overexpression significantly decreased ROS accumulation, while downregulation of YAP expression increased ROS accumulation. In summary, our results demonstrate that the Hippo/YAP signaling pathway plays an important role in reducing HC injury and maintaining auditory function after aminoglycoside exposure. YAP overexpression could protect against neomycin-induced HC loss by inhibiting C-Ablmediated cell apoptosis and decreasing ROS accumulation, suggesting that YAP could be a novel therapeutic target for aminoglycosides-induced sensorineural hearing loss in the clinic.
\end{abstract}

Keywords Aminoglycosides $\cdot$ Hair cells $\cdot$ Apoptosis $\cdot$ Protection $\cdot$ Hippo/YAP signaling pathway $\cdot$ Sensorineural hearing loss (SNHL)

\section{Introduction}

Hearing loss is one of the most common sensory defects in humans. According to the World Health Organization (WHO) report in 2018, approximately 466 million people

Maohua Wang, Ying Dong and Song Gao contributed equally to this work.

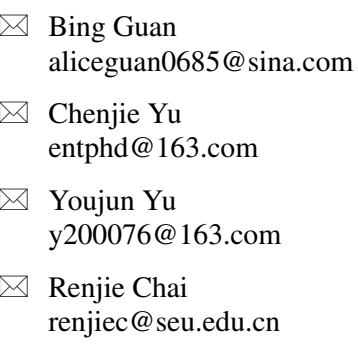

Extended author information available on the last page of the article suffer from hearing loss worldwide [1]. There are three main types of hearing loss-sensorineural hearing loss, conductive hearing loss, and mixed hearing loss-of which sensorineural hearing loss accounts for the vast majority of cases [2]. While noise exposure, aging, long-term use of ototoxic drugs [3], and viral infection can lead to varying degrees of sensorineural hearing loss, irreversible damage to cochlear hair cells (HCs) is the fundamental cause of sensorineural hearing loss $[4,5]$. More than 150 drugs have ototoxic effects $[6,7]$, and aminoglycoside antibiotics are one of the most common ototoxic drugs causing sensorineural hearing loss in the clinic [8]. HCs sense the stimulation of sound waves, and mammalian HCs lack regenerative capacity; thus, once HCs are damaged, permanent hearing loss is inevitable.

The Hippo signaling pathway was first discovered in the study of the Drosophila Warts (WTS) gene mutation in 1995 [9]. Later studies found that mutations of Salvador (SAV) [10], Hippo (HPO) [11], and Mob as tumor suppressor 
(MATS) [12] could also cause excessive growth of tissues and organs. Given their close relationship, these genes are collectively referred to as the Hippo signaling pathway [13]. In 2005, Yorkie (YKI), a major effector factor, was found to play a regulatory role in the Hippo signaling pathway, and conditional knockout of YKI was found to inhibit tissue overgrowth caused by mutations of upstream effectors such as WTS, SAV, and HPO [14]. Similarly, the Hippo signaling pathway is highly conserved in mammals, and its core effector factors include Mammalian ste20-like protein kinase 1/2 (MST1/2) (same as HPO), large tumor suppressor kinase 1/2 (LATS1/2) (same as WTS), and Yes-associated protein (YAP) (same as YKI) [15]. As a core effector of the Hippo signaling pathway in mammals, conditional knockout of YAP can also inhibit cell overgrowth [16]. As the transcriptional coactivator, YAP lacks a domain that can directly bind to DNA, so it needs to be combined with downstream transcription factors to enter the nucleus and carry out its biological functions [17]. Tea domain transcription factor (TEAD), a major transcription factor, interacts with YAP and plays a regulatory role [18].

The Hippo/YAP signaling pathway has been shown to be able to maintain organ size and homeostasis of the internal environment by regulating cell proliferation, differentiation, and apoptosis, and it is known to play an important role in the occurrence and development of cancer [15, 19-23]. There are three regulatory mechanisms of YAP that are regulated by Hippo signaling, namely phosphorylation [24], protein-protein interactions [25], and the competitive binding of VGLL4 to the TEAD transcription factor [26, 27]. When the Hippo signaling pathway is on, activated MST1/2 phosphorylates LATS1/2, leading to the phosphorylation of YAP, which then either stays in the cytoplasm or degrades [13] and participates in cell apoptosis and differentiation [16]. In contrast, when the Hippo signaling pathway is off, YAP enters the nucleus after dephosphorylation and forms a complex with TEAD to participate in cell proliferation [28]. In addition to numerous upstream factors regulating the Hippo signaling pathway, the cascade itself has a negative feedback regulation system. YAP can increase the expression of NF2, LAST2, and MST1 by binding to TEAD in the nucleus, which has a negative feedback regulatory role on YAP [13].

Recently, an increasing number of scholars have found that YAP activation plays an important role in organ regeneration and regenerative medicine [29]. Indeed, YAP activation can promote the proliferation of retinal precursor cells and the differentiation of pigment cells. In contrast, conditional knockout of YAP could reduce cell proliferation, promote cell apoptosis, and induce pigment deposition leading to retinal degeneration in mice [30], indicating that the Hippo/YAP signaling pathway plays a critical regulatory role in the development of the retina. Other studies have also found that YAP knockout not only inhibits bile duct proliferation but also enhances hepatocyte necrosis and inhibits hepatocyte proliferation, while YAP activation prevents cholestasis injury in mice [31]. The loss of YAP in the nervous system further aggravates lysophosphatidic acid (LPA)-induced hemorrhagic hydrocephalus, which is partially recovered after enhanced YAP expression [32]. Upregulation of YAP expression has also been found to promote the healing of skin wounds [33]. Moreover, enhanced YAP activity can protect the myocardium in acute stress injury [34]. In kidney disease, podocyte injury leads to podocyte death and loss, which results in progressive kidney damage and ultimately kidney failure, and the loss of YAP in podocytes further increases Adriamycin-induced podocyte apoptosis [35]. All of these studies have demonstrated the regulatory role of the Hippo/YAP signaling pathway in tissue protection and regeneration after injury. However, the role of the Hippo/YAP signaling pathway in protecting HCs against damage in the mouse cochlea remains unclear.

In this study, we used the YAP agonist XMU-MP-1 (XMU) and the YAP inhibitor Verteporfin (VP) to regulate the expression level of YAP and investigated the role of the Hippo/YAP signaling pathway in protecting against aminoglycoside-induced cochlear $\mathrm{HC}$ damage in vitro. We found that the Hippo/YAP signaling pathway could regulate C-Abl-mediated HC apoptosis and the accumulation of ROS, which protects against neomycin-induced $\mathrm{HC}$ loss after neomycin injury. Thus, aminoglycoside-induced cochlear HC damage could be prevented by regulating the Hippo/YAP signaling pathway. This will allow hearing function to be preserved and provide a novel therapeutic target for aminoglycoside-induced sensorineural hearing loss.

\section{Materials and methods}

\section{Experimental animals}

In this study, we used wild-type (WT) FVB mice from the Jackson Laboratory. The mice were raised and provided by the Key Laboratory of "Development and Disease-related Genes," Ministry of Education, Southeast University. FVB mice are inbred WT mice that have the following characteristics: uniform genetic background, good consistency of experimental results, strong reproductive capacity, large litter size, and low spontaneous tumor rate. Therefore, inbred mice are preferred as experimental animals to study gene function or disease mechanisms. All animal procedures were performed according to the protocols approved by the Animal Care and Use Committee of Southeast University, and were consistent with the National Institutes of Health Guide for the Care and Use of Laboratory Animals. All efforts were 
made to reduce the number of animals used and to minimize their suffering.

\section{Tissue culture}

Cochleae were dissected from postnatal day (P)3 mice and cultured as previously reported [36]. The cochlear sensory epithelium was isolated and seeded intact on a glass coverslip coated with Cell-Tak (Corning, 354240) and cultured in DMEM/F12 (Gibco, 11330-032) supplemented with 2\% B27 (Invitrogen, 17504-044), 1\% N-2 (Invitrogen, 17502048), and $50 \mathrm{mg} / \mathrm{ml}$ ampicillin (Sigma-Aldrich, P0781) [37]. In the experimental group, the cochlear tissues were pretreated with $2 \mu \mathrm{M}$ VP (dissolved in DMSO, SigmaAldrich, SML0534-5ML) or $1 \mu \mathrm{M}$ XMU (dissolved in DMSO, Astatech, 43245) for $12 \mathrm{~h}$. Next, $0.5 \mathrm{mM}$ neomycin (Sigma-Aldrich, N6386-5G) was added for $12 \mathrm{~h}$ to induce $\mathrm{HC}$ damage. After neomycin was removed, the tissues were allowed to recover in serum-free medium for a further $12 \mathrm{~h}$. In the control group, the tissues were recovered in serumfree medium for $36 \mathrm{~h}$ without neomycin, XMU, or VP, and the medium was changed every $12 \mathrm{~h}$.

\section{Immunofluorescence}

The TUNEL BrightRed Apoptosis Detection Kit (Vazyme, A111-03), Anti-Cleaved Caspase-3 antibody (Cell Signaling Technology, 9664S), Mito-SOX Red (Life Technologies, M36008), Anti-YAP antibody (Cell Signaling Technology, 12395S, 1:400 dilution), Anti-C-Abl antibody (Affinity Biosciences, AF6038, 1:400 dilution), Anti-Myosin7a antibody (Proteus Biosciences, 25-6790, 1:1000 dilution), Anti-Sox 2 antibody (RD, AF-2018, 1:400 dilution), Alexa Fluor 488 donkey Anti-Rabbit IgG (Invitrogen, A-21206, 1:400 dilution), Alexa Fluor 555 donkey Anti-Mouse IgG (Invitrogen, A-31570, 1:400 dilution), Alexa Fluor 647 donkey Anti-Goat IgG (Invitrogen, A-21447, 1:400 dilution), and DAPI (Solarbio, C0060, 1:1000 dilution) were used to measure apoptotic cells and to stain HCs and nuclei. The dissected cochlear tissues were fixed in $4 \%$ paraformaldehyde (Sigma-Aldrich, 158127) for $1 \mathrm{~h}$, then washed three times with PBST $(1 \times$ PBS [Sigma, P5493] with $0.1 \%$ Triton X-100 (Solarbio, 1109F0521). After blocking for $1 \mathrm{~h}$ in medium (10\% donkey serum, $0.1 \%$ Triton $\mathrm{X}-100$, and $1 \%$ BSA in PBS at pH 7.2) at room temperature, the samples were incubated with primary antibody diluted in PBT-1 (0.1\% Triton X-100, 5\% donkey serum, $1 \%$ BSA in PBS at $\mathrm{pH}$ 7.2) overnight at $4{ }^{\circ} \mathrm{C}$. The samples were washed three times with PBST and then incubated with the secondary antibody diluted in PBT-2 (1\% BSA and 0.1\% Triton X-100 in PBS at $\mathrm{pH}$ 7.2) for $1 \mathrm{~h}$ at room temperature. After incubating with secondary antibody, the samples were washed three times with PBST and mounted on glass slides with
DAKO (DAKO, S3023). Finally, the fluorescence images were obtained by a confocal microscope (LSM710, Zeiss, Heidelberg).

\section{Western blot}

The dissected cochlear tissues were lysed with RIPA Lysis Buffer (Protein Biotechnology, PP109) plus Phosphatase Inhibitor Cocktails (Roche, 04693132001). The fully reacted tissue mixture was centrifuged at $13,000 \mathrm{rpm}$ for 5-10 min at $4{ }^{\circ} \mathrm{C}$. The supernatant solution was mixed with $5 \times$ sodium dodecyl sulfate buffer (Beyotime, P0015L) at a ratio of 1:4 to obtain a protein sample, and the samples were boiled in water for $15 \mathrm{~min}$. Equal amounts of protein samples were loaded onto a $10 \%$ Tris-glycine SDS-PAGE gel, electrophoresed at $120 \mathrm{~V}$ for $2 \mathrm{~h}$, and then transferred to a $0.2-\mu \mathrm{m}$ polyvinylidene difluoride membrane (Millipore, Immobilon ISEQ00010). Following transfer, the membranes were blocked with 5\% BSA (Biosharp, 4240), then washed three times with $1 \times$ PBST $(1 \times$ PBS [Sigma, P5493] with $0.1 \%$ Triton X-100 [Solarbio, 1109F0521]). Protein concentrations were measured using a BCA Protein Quantification Kit (Protein Biotechnology, PP202), with GAPDH as the reference protein. Anti-YAP mouse polyclonal antibody (Cell Signaling Technology, 12395S, 1:400 dilution), Anti-C-Abl rabbit polyclonal antibody (Affinity Biosciences, AF6038, 1:400 dilution), and GAPDH mouse monoclonal antibody (Abcam, ab8245, 1:1000 dilution) were used as primary antibodies. Horseradish peroxidase-conjugated goat antirabbit (or anti-mouse) IgG (Abcam, ab6789, ab6721) was used as the secondary antibody. The protein signals were detected using a SuperSignal West Dura chemiluminescent substrate kit (Thermo Fisher Scientific, 34075) on a FluorChem M system (ProteinSimple, San Jose, CA, USA).

\section{Quantitative real-time PCR (qPCR)}

Total RNA was extracted from 8 to 10 whole cochleae using TRIzol Reagent (Protein Biotechnology, PR910). The integrity of the RNA samples was evaluated by the A260/ A280 ratio, and the samples were reverse transcribed to cDNA using the Revert Aid First Strand cDNA Synthesis kit (Thermo Fisher Scientific, K1622). The qPCR was performed on an Applied Biosystems CFX96 real-time PCR system (Bio-Rad, Hercules, CA, USA) using the FastStart Universal SYBR Green (Rox) qRT-PCR Master Mix (Roche Life Science, 4913850001). The qRT-PCR conditions were set as follows: $15 \mathrm{~s}$ denaturation at $95{ }^{\circ} \mathrm{C}$ followed by 40 cycles of denaturation at $95{ }^{\circ} \mathrm{C}$ for $15 \mathrm{~s}$, annealing at $60{ }^{\circ} \mathrm{C}$ for $60 \mathrm{~s}$, and extension at $72{ }^{\circ} \mathrm{C}$ for $20 \mathrm{~s}$. $\beta$-actin was used as the housekeeping gene for control. The concentration and purity of RNA and cDNA were determined with a 
Nano-Drop (Thermo Fisher, 2000). The primer sequences are shown in Table 1.

\section{Cell counts}

In the neomycin-treated groups, we counted the number of Myosin $7 \mathrm{a}+\mathrm{HCs}$ that remained under a $40 \times$ microscope. The same procedure was used to quantify cleaved caspase-3 + /Myosin 7a + cells, TUNEL + /Myosin 7a + cells, and MitoSOX Red +/Myosin 7a + cells. In all experiments, only one cochlea from each mouse was used for immunofluorescence and quantification; therefore, $\mathrm{n}$ represents the number of mice examined.

\section{Statistical analysis}

Data are shown as the mean \pm the standard error of the mean (S.E.M). Statistical analyses were conducted using Microsoft Excel and GraphPad Prism 7 software. The counting data and qPCR data were statistically analyzed by GraphPad Prism 7, while ImageJ was used to count the number of cells in the immunofluorescence map, where the image size, layer, and contrast could be modified. Two-tailed, unpaired Student's $t$ tests were used to determine statistical significance when comparing two groups, and one-way ANOVA followed by a Dunnett multiple comparison test was used when comparing more than two groups. $p$ values $<0.05$ were considered statistically significant. The experimental data and images were recombined and typeset using Adobe Illustrator software. Each experiment was repeated at least three times to ensure the accuracy and reliability of the experimental results $(n \geq 3)$. The number of replicates is indicated in each figure legend.
Fig. 1 The expression and localization of YAP in mouse cochlear HCs. A The cochleae were dissected from P3 WT mice, and immunolabeled with Myosin7a (green), Sox 2 (blue), and YAP (red). Immunofluorescence staining showed that the expression and localization of YAP in the P3 WT mouse cochlea under a $5 \times$ microscope. B Western blot results showed that YAP was strongly expressed in the mouse cochlea and brain. C Immunofluorescence staining showed the expression and localization of YAP in the P3 WT mouse cochlea under a $63 \times$ microscopy. D Immunofluorescence staining showed the expression and localization of YAP in the P30 WT mouse cochlea under a $63 \times$ microscope. C, D Immunofluorescence staining showed that YAP was expressed in the cytoplasm of cochlear HCs. E RTPCR results showed that YAP was strongly expressed in the mouse cochlea and brain. $H C$ hair cell, $S C$ supporting cell; Scale bar: A: $200 \mu \mathrm{m} ; \mathbf{C}, \mathbf{D}: 10 \mu \mathrm{m} . N=5$

\section{Results}

\section{Expression and localization of YAP in mouse cochlear HCs}

To determine the role of the Hippo/YAP signaling pathway in cochlear HC injury protection, we first studied the expression and localization of YAP (a core effector of Hippo signaling pathway) in the mouse cochlea. We dissected the cochleae from postnatal day (P) 3 and P30 WT mice and immunolabeled them with the HC marker Myosin7a, the supporting cell (SC) marker Sox2, and DAPI to label the DNA. We first observed the cochlear basilar membrane under a $5 \times$ microscope (Fig. 1A). Immunofluorescence staining showed that YAP was expressed in the cytoplasm of cochlear HCs (Fig. 1C, D). Western blot and RT-PCR demonstrated that YAP was strongly expressed in the P3 mouse cochlea and brain (Fig. 1B, E). These results suggested that
Table 1 The primer sequences were used in this study

\begin{tabular}{|c|c|c|}
\hline Gene & Forward sequence $\left(5^{\prime}-3^{\prime}\right)$ & Reverse sequence $\left(5^{\prime}-3^{\prime}\right)$ \\
\hline$Y A P$ & ACCCTCGTTTTGCCATGAAC & TGTGCTGGGATTGATATTCCGTA \\
\hline$C-A b l$ & AGCCGCTTCAACACTCTGG & ACACCGTAGATAGTGGGCTTG \\
\hline Bax & TGAAGACAGGGGCCTTTTTG & AATTCGCCGGAGACACTCG \\
\hline Caspase8 & ATGGCGGAACTGTGTGACTCG & GTCACCGTGGGATAGGATACAGCA \\
\hline Apaf1 & AGTGGCAAGGACACAGATGG & GGCTTCCGCAGCTAACACA \\
\hline$F A D D$ & GCTCCAGAATGGGCGAAGTAA & ACGGATGTGCGGAGGTAAAAA \\
\hline$B c l-2$ & GTCGCTACCGTCGTGACTTC & CAGACATGCACCTACCCAGC \\
\hline$T E A D-2$ & GAAGACGAGAACGCGAAAGC & GATGAGCTGTGCCGAAGACA \\
\hline Lats1 & AAAGCCAGAAGGGTACAGACA & CCTCAGGGATTCTCGGATCTC \\
\hline$P 73$ & GCACCTACTTTGACCTCCCC & GCACTGCTGAGCAAATTGAAC \\
\hline$\beta$-actin & ACGGCCAGGTCATCACTATTG & AGGGGCCGGACTCATCGTA \\
\hline Nqol & AGGATGGGAGGTACTCGAATC & AGGCGTCCTTCCTTATATGCTA \\
\hline Gsr & TGCACTTCCCGGTAGGAAAC & GATCGCAACTGGGGTGAGAA \\
\hline Glrx & AGTCTGGAAAGGTGGTCGTG & CCATTAGCATGGCTGGACGA \\
\hline Sodl & GGAGCAAGGTCGCTTACAGA & AGTGACAGCGTCCAAGCAAT \\
\hline Alox15 & GGCTCCAACAACGAGGTCTAC & AGGTATTCTGACACATCCACCTT \\
\hline
\end{tabular}


A

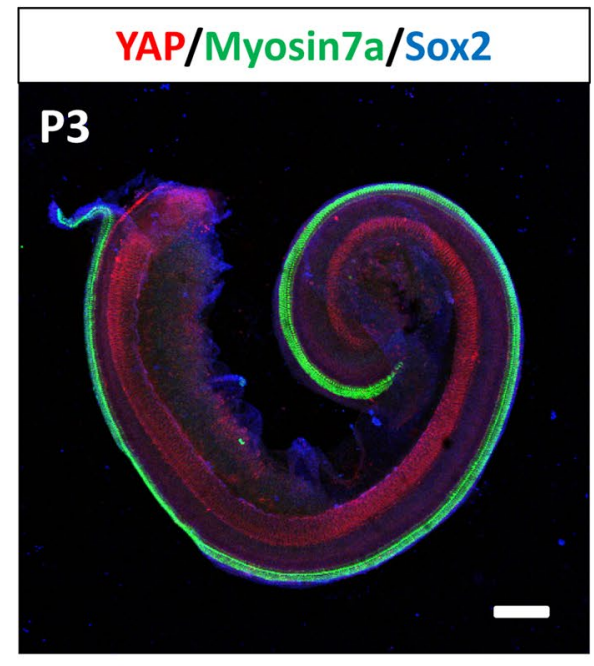

c

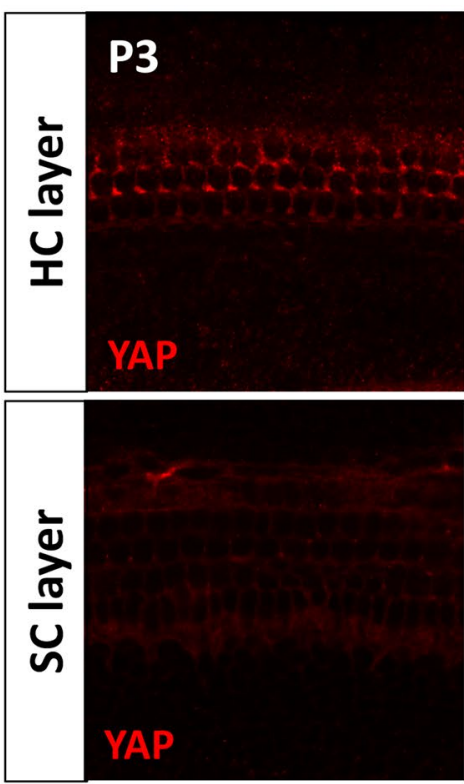

D
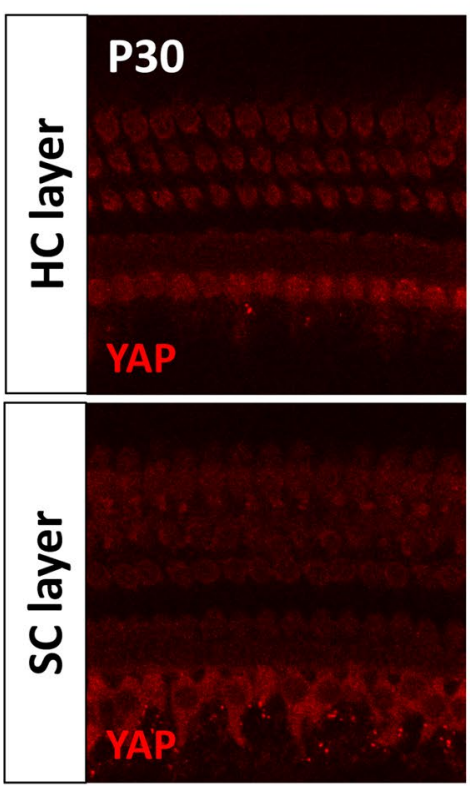

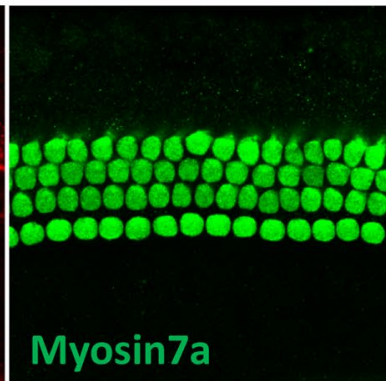

Sox2

E

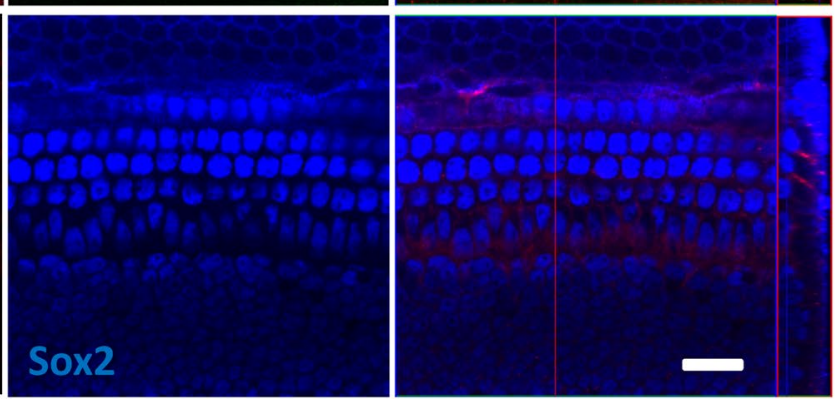

B

conder

YAP

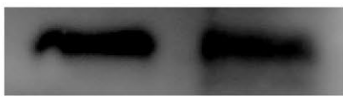

75kD

GAPDH

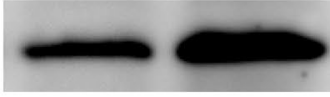

$36 \mathrm{kD}$

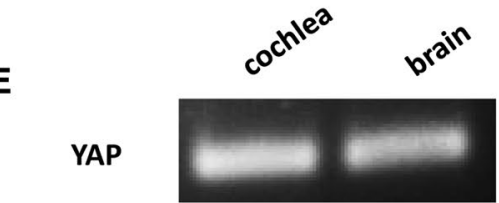

GAPDH
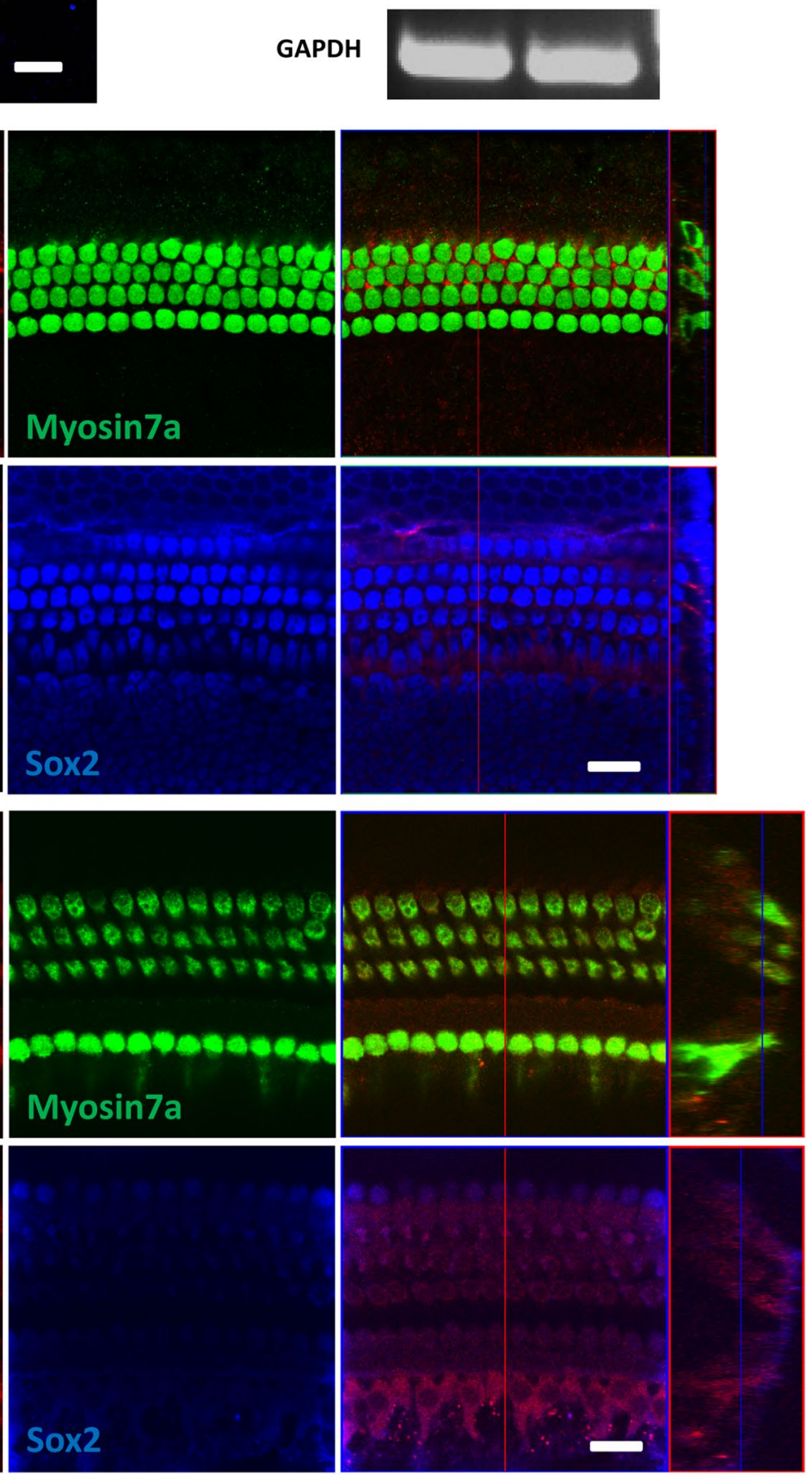
YAP plays an important role in the development of $\mathrm{HCs}$ in the mouse cochlea, and may be involved in the regulation of related physiological processes in the inner ear, which provides a basis for further study.

\section{The Hippo pathway is activated and the expression of YAP is decreased in cochlear HCs after neomycin treatment}

We next explored the expression of YAP and Hippo target genes in the cochlear HCs after neomycin treatment. We dissected the cochleae from P3 WT mice and cultured them with $0.5 \mathrm{mM}$ neomycin for $12 \mathrm{~h}$ (Fig. 2A). Both immunofluorescence and western blot results showed that the expression of YAP was decreased in the HCs after neomycin injury (Fig. 2B, D). qPCR results revealed that the expressions of YAP and the Hippo downstream target gene TEAD-2 were significantly downregulated after neomycin treatment (Fig. 2C). These results demonstrated that the Hippo pathway was activated in the cochlear HCs after neomycin injury, indicating that the Hippo/YAP signaling pathway is a protective physiological mechanism against neomycin injury.

\section{YAP overexpression protects against neomycin-induced HC loss in vitro}

To investigate the protective role of the Hippo/YAP signaling pathway against neomycin-induced $\mathrm{HC}$ loss, we used the YAP agonist XMU and the YAP inhibitor VP in two independent in vitro experiments. First, cochlear tissues from P3 WT mice were cultured with XMU $(5 \mu \mathrm{M})$ or VP $(5 \mu \mathrm{M})$ for $36 \mathrm{~h}$ (Fig. 2A). Immunofluorescence staining demonstrated upregulated expression of YAP in the XMU-treated group and downregulated expression of YAP in the VP-treated group. The HCs were lost after VP treatment (Fig. 2B). Both $\mathrm{XMU}$ and VP dissolve in DMSO, and it was necessary to find an appropriate drug concentration because excessive concentrations are cytotoxic and will lead to cell apoptosis, which will affect the experimental results. To explore the optimal concentration of XMU and VP, we dissected the cochleae from P3 WT mice and cultured them with different concentrations of XMU and VP for $36 \mathrm{~h}$ (Fig. 2A). qPCR results showed that XMU-treated groups had significantly higher expression of YAP and the Hippo downstream target gene TEAD-2 than the control group, while the VPtreated group had significantly lower expression of YAP and TEAD-2 than the control group. Compared to $2 \mu \mathrm{M}$ and $5 \mu \mathrm{M}$, the optimal concentration of XMU was $1 \mu \mathrm{M}$, and the optimal concentration of VP was $2 \mu \mathrm{M}$ compared to $1 \mu \mathrm{M}$ and $5 \mu \mathrm{M}$. Therefore, we chose $1 \mu \mathrm{M}$ XMU and $2 \mu \mathrm{M}$ VP pretreatment for $12 \mathrm{~h}$ under the treatment conditions in the following experiments (Fig. 3B, C).

We next used $1 \mu \mathrm{M}$ XMU or $2 \mu \mathrm{M}$ VP to pretreat the cochleae for $12 \mathrm{~h}$ before neomycin exposure. We then treated the cultured cochleae with $0.5 \mathrm{mM}$ neomycin together with $1 \mu \mathrm{M}$ XMU or $2 \mu \mathrm{M}$ VP for the next $12 \mathrm{~h}$. Finally, we removed neomycin and treated the cultured cochleae with $1 \mu \mathrm{M}$ XMU or $2 \mu \mathrm{M}$ VP for another $12 \mathrm{~h}$ (Fig. 3A). The cochleae were immunolabeled with the $\mathrm{HC}$ marker Myosin7a and the DNA marker DAPI after culturing the cochleae with different treatments. Immunofluorescence staining showed that the XMU/neomycin-treated group had significantly less HC loss than the neomycin-only group. In contrast, the VP/neomycin-treated group had significantly greater HC loss than the neomycin-only group (Fig. 3D, E). These results demonstrate that YAP overexpression could protect against neomycin-induced $\mathrm{HC}$ loss in vitro.

\section{The Hippo/YAP signaling pathway regulates neomycin-induced HC apoptosis in vitro}

We also sought to determine the mechanism by which the Hippo/YAP signaling pathway protects against neomycininduced HC loss. Previous studies have reported that neomycin kills HCs by inducing apoptosis, in which TUNEL and cleaved-caspase 3 were used as markers of aminoglycoside-induced $\mathrm{HC}$ apoptosis [38-41]. Therefore, immunofluorescence staining with TUNEL and cleaved-caspase-3 was performed to detect apoptotic cochlear HCs after different treatments. The immunofluorescence results showed that the numbers of cleaved-caspase $3+/$ Myosin $7 \mathrm{a}+$ and TUNEL +/Myosin7a + double-positive cells per $100 \mathrm{~mm}$ of the cochlea in the middle turn were significantly increased in the neomycin-treated group compared to the control group (Fig. 4A-D). In additon, the numbers of cleaved-caspase $3+/$ Myosin7a + and TUNEL +/Myosin7a + double-positive cells in the XMU/neomycin-treated group were significantly reduced compared to those in the neomycin-only group. In contrast, when cochleae were pretreated with VP the numbers of cleaved-caspase $3+/$ Myosin $7 \mathrm{a}+$ and TUNEL + / Myosin $7 \mathrm{a}+$ double-positive cells were increased compared to those in the neomycin-only group (Fig. 4A-D).

We next performed qPCR to explore the expression level of apoptosis-related genes in the cochlea after different treatments. The qPCR results demonstrated that the expression of the pro-apoptotic genes Caspase8, Bax (Bcl-2-associated X protein), and Apafl (Apoptotic Peptidase Activating Factor 1) was significantly increased after neomycin treatment compared to that of the control group, and the expression of the anti-apoptotic gene $B c l-2$ (B Cell Leukemia/Lymphoma 2) was significantly decreased compared to that in the control group (Fig. 4E). Furthermore, the YAP inhibitor VP significantly upregulated the expression of the pro-apoptotic 

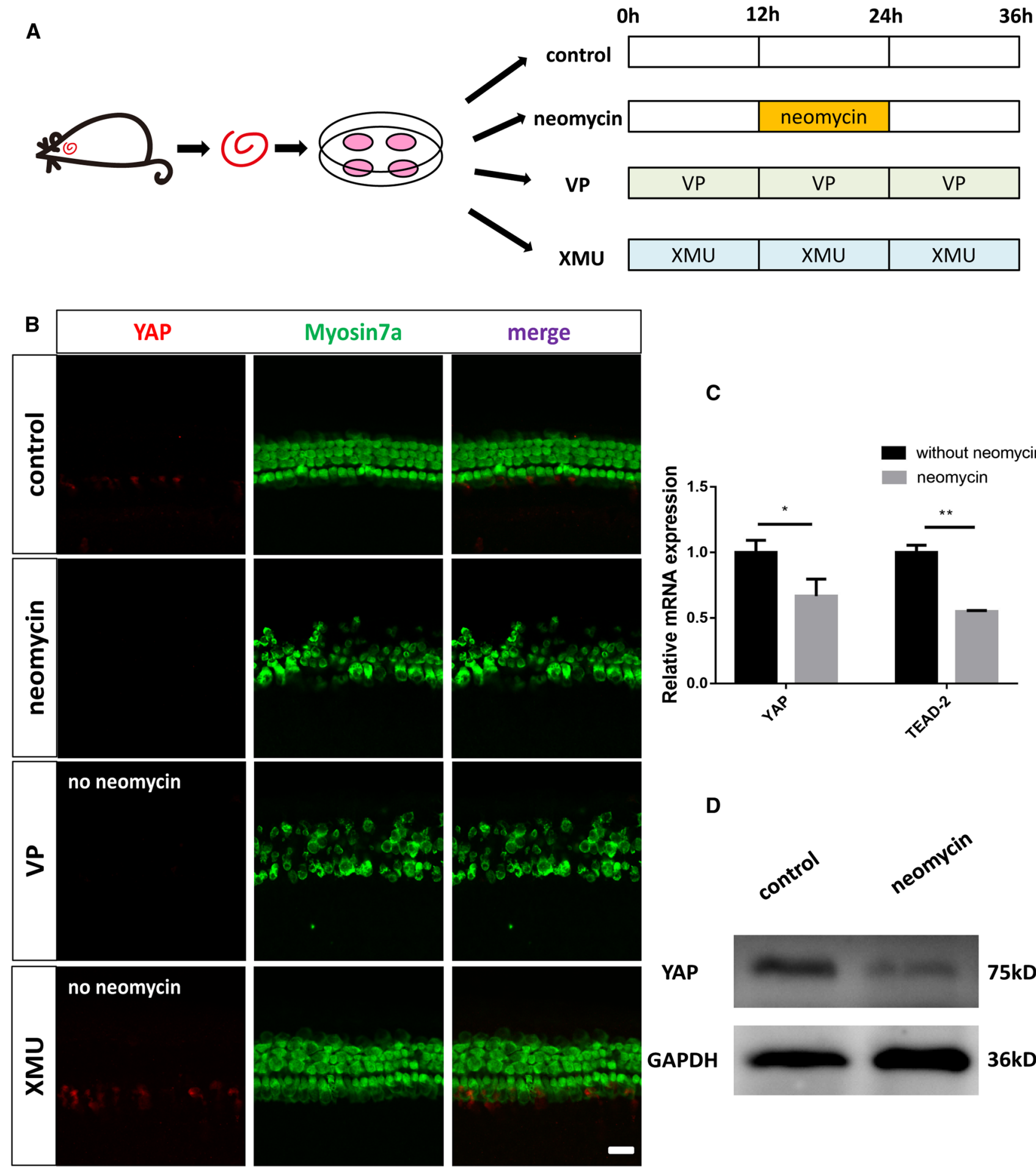

Fig. 2 The Hippo pathway is activated and the expression of YAP is decreased in cochlear HCs after neomycin treatment. A The dissected cochleae were cultured in vitro with different treatments. In the neomycin-treated group, the cochleae were allowed to recover for the first $12 \mathrm{~h}$, treated with $0.5 \mathrm{mM}$ Neomycin for the next $12 \mathrm{~h}$, and then allowed to recover again for another $12 \mathrm{~h}$. In the VP-treated group, the cochleae were cultured with $5 \mu \mathrm{M}$ VP for $36 \mathrm{~h}$ without neomycin. In the XMU-treated group, the cochleae were cultured with $5 \mu \mathrm{M}$ $\mathrm{XMU}$ for $36 \mathrm{~h}$ without neomycin. B Immunofluorescence staining showed that the expression level of YAP was decreased in the HCs

after neomycin treatment compared with the control group and that the expression of YAP was upregulated in the XMU-treated group and downregulated in the VP-treated group. The HCs were lost after VP treatment. C qPCR results revealed that the expression of YAP and the Hippo downstream target gene TEAD-2 was significantly downregulated after neomycin treatment. D Western blot results showed that the expression of YAP was decreased in the HCs after neomycin injury. ${ }^{*} p<0.05,{ }^{*} p<0.01, * * * p<0.001, n=3$. Scale bars $=20 \mu \mathrm{m}$ 


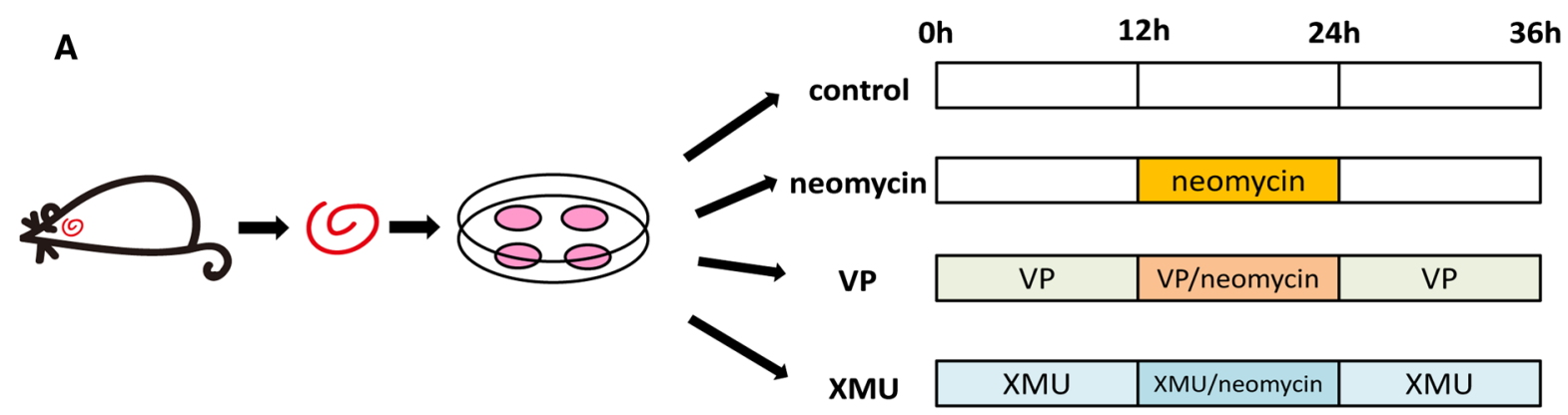

B

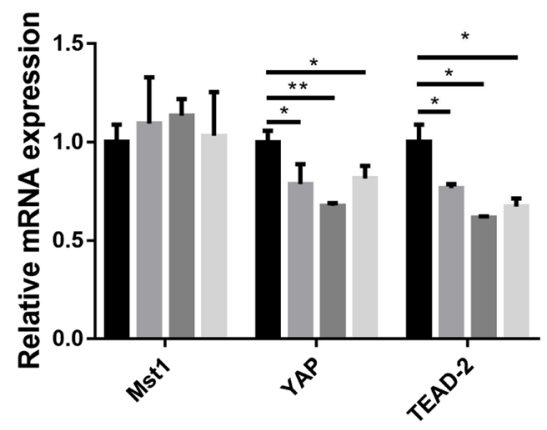

C

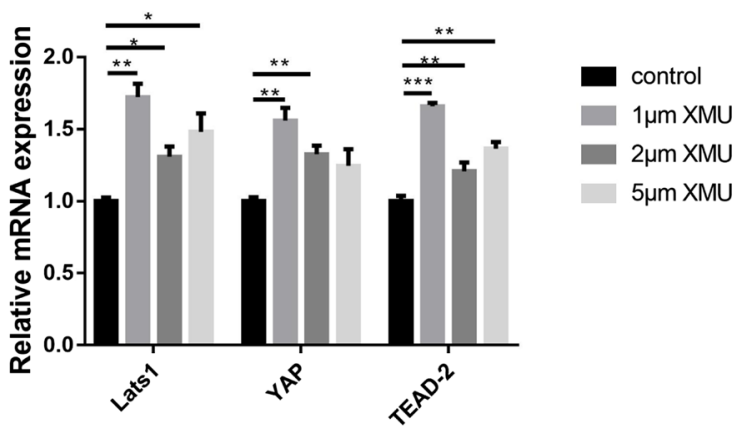

D

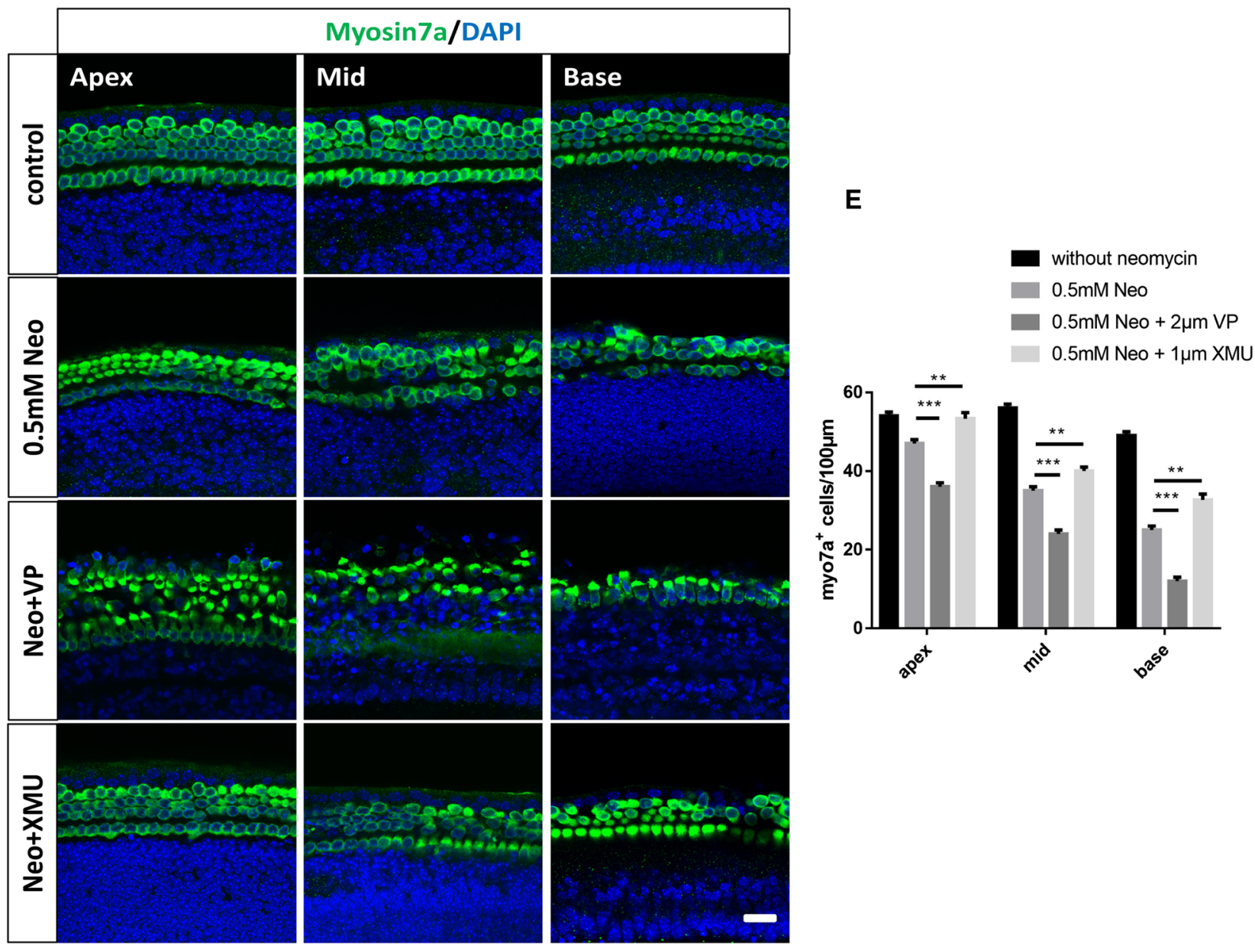


4Fig. 3 YAP overexpression protects against neomycin-induced HC loss in vitro. A Schematic diagram of drug addition in tissue culture (divided into four groups). B, C The mRNA levels of YAP were analyzed by qPCR after culturing with different concentrations of XMU/ VP under the same conditions. The results showed that the optimal concentration of XMU was $1 \mu \mathrm{M}$ and the optimal concentration of $\mathrm{VP}$ was $2 \mu \mathrm{M}$. D Immunofluorescence staining with Myosin7a and DAPI in the apical, middle, and basal turns of the cochlear basilar membrane after different treatments. E Quantification of the numbers of Myosin7a + / DAPI + double-positive cells in D showing that the XMU-treated group had significantly reduced HC loss after neomycin treatment. $* p<0.05, * * p<0.01, * * * p<0.001, n=4$. Scale bars $=20 \mu \mathrm{m}$

genes Caspase8, Bax, and Apaf1, and reduced the expression of the anti-apoptotic gene $B c l-2$. The expression of the pro-apoptotic genes Caspase8, Bax, and Apafl was significantly decreased, and the expression of the anti-apoptotic gene $\mathrm{Bcl}$-2 was increased when the cochleae were treated with the YAP agonist XMU (Fig. 4F). Taken together, the above results suggested that the Hippo/YAP signaling pathway could regulate neomycin-induced $\mathrm{HC}$ apoptosis in vitro.

\section{The Hippo/YAP signaling pathway regulates the ROS levels in cochlear HCs after neomycin injury}

Previous studies have reported that aminoglycosideinduced accumulation of ROS is closely related to HC apoptosis in the mouse cochlea [42-44]. Mito-SOX Red is a redox fluorophore that selectively detects mitochondrial superoxide and can be used to evaluate the ROS levels in different treatment groups [45-48]. To determine the relationship between $\mathrm{HC}$ loss and oxidative stress in the mouse cochlea, we dissected and cultured the cochleae from P3 WT mice and then treated them with neomycin together with XMU or VP (Fig. 5A). We used Mito-SOX Red to detect the ROS levels in cochlear HCs by immunofluorescence staining. The immunofluorescence results showed that the number of Mito-SOX +/Myosin7a + double-positive cells per $100 \mathrm{~mm}$ of the cochlea in the middle turn was significantly increased in the neomycin-treated group compared to that in the control group (Fig. 5B, C). Additionally, the number of Mito-SOX +/Myosin 7a + doublepositive cells in the XMU/neomycin-treated group was significantly less than that in the neomycin-treated group. In contrast, when the cochleae were pretreated with VP, the number of Mito-SOX +/Myosin 7a + double-positive cells was increased compared to that in the neomycin-treated group (Fig. 5B, C). We next conducted qPCR to investigate the expression level of redox-related genes in the mouse cochlea after different treatments. We found that the expressions of the antioxidant genes Nqol (NAD (P) H: Quinone Oxidoreductase 1), Gsr (Glutathione reductase), Glrs (Glutaredoxin), and Sodl (Superoxide dismutase 1) were significantly decreased after neomycin treatment compared to the control group, and the expression of the pro-oxidant gene Alox15 (Arachidonate 15-Lipoxygenase) was significantly increased in the neomycin group compared to the control group (Fig. 5D). In addition, the expression of the antioxidant genes Nqo1, Gsr, Glrs, and Sodl was significantly increased, and the expression of the pro-oxidant gene Alox 15 was decreased when the cochleae were pretreated with XMU compared to the neomycin group (Fig. 5D). These results demonstrated that the Hippo/YAP signaling pathway regulates the accumulation of ROS in cochlear HCs after neomycin injury.

\section{C-Abl expression is regulated by the Hippo/YAP signaling pathway in cochlear HCs after neomycin exposure}

It has been reported that the Hippo/YAP signaling pathway prevents DNA damage-induced apoptosis through inhibition of the non-receptor tyrosine kinase C-Abl (Abelson murine leukemia viral oncogene) [49]. YAP activates pro-apoptotic genes along with $\mathrm{p} 73$ under conditions of DNA damage, and $\mathrm{C}-\mathrm{Abl}$ promotes the association of YAP with $\mathrm{p} 73$, which induces apoptosis [50]. This program switching is mediated by C-Abl via phosphorylation of YAP at the Y357 residue $[51,52]$. To investigate the relationship between $\mathrm{C}-\mathrm{Abl}$ expression and neomycin-induced $\mathrm{HC}$ apoptosis in the mouse cochlea, we first verified the expression and localization of C-Abl by immunofluorescence staining, which showed that $\mathrm{C}$-Abl was expressed in the nuclei of cochlear HCs (Fig. 6A). We next investigated the C-Abl expression in neomycin-treated cochleae and XMU/neomycin-treated cochleae. After $12 \mathrm{~h}$ of neomycin treatment, intense nuclear $\mathrm{C}$-Abl staining was observed in neomycin-treated cochleae compared to the control cochleae, indicating active $\mathrm{C}-\mathrm{Abl}$ signaling in response to neomycin exposure (Fig. 6B, C). Moreover, the immunofluorescence staining intensity of $\mathrm{C}-\mathrm{Abl}$ was significantly decreased in XMU/neomycintreated cochleae compared to neomycin-treated cochleae (Fig. 6B, C). qPCR and western blot results showed that $\mathrm{C}$-Abl expression was significantly increased in neomycintreated cochleae compared to control cochleae, and was significantly decreased in XMU/neomycin-treated cochleae compared to neomycin-treated cochleae (Fig. 7B-D). These results suggest that $\mathrm{C}$ - $\mathrm{Abl}$ expression is increased in cochlear $\mathrm{HCs}$ after neomycin exposure and that $\mathrm{C}$-Abl expression in cochlear $\mathrm{HCs}$ is regulated by the Hippo/YAP signaling pathway.

\section{YAP overexpression inhibits C-Abl-mediated HC apoptosis in cochlear HCs after neomycin damage}

Our initial findings suggested that C-Abl plays an important role in neomycin-induced HC apoptosis. To confirm 
A

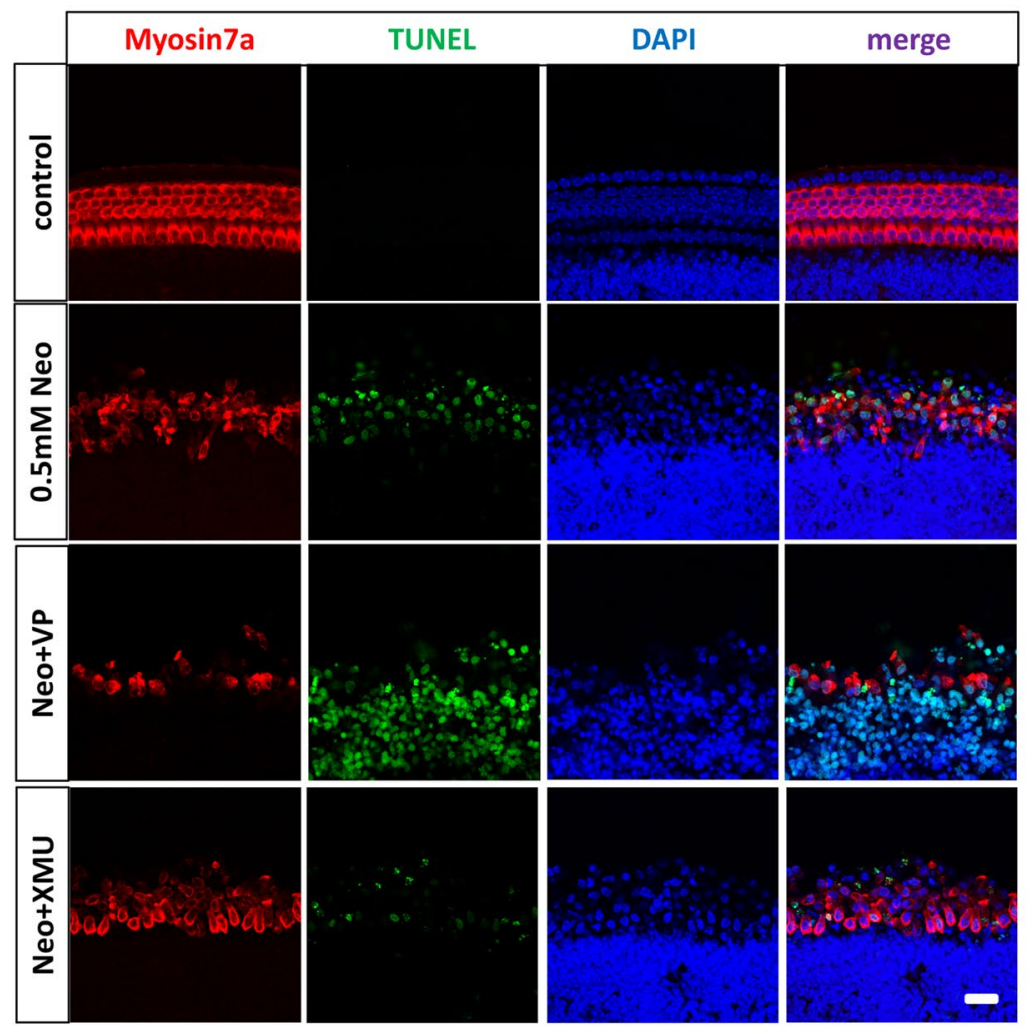

C

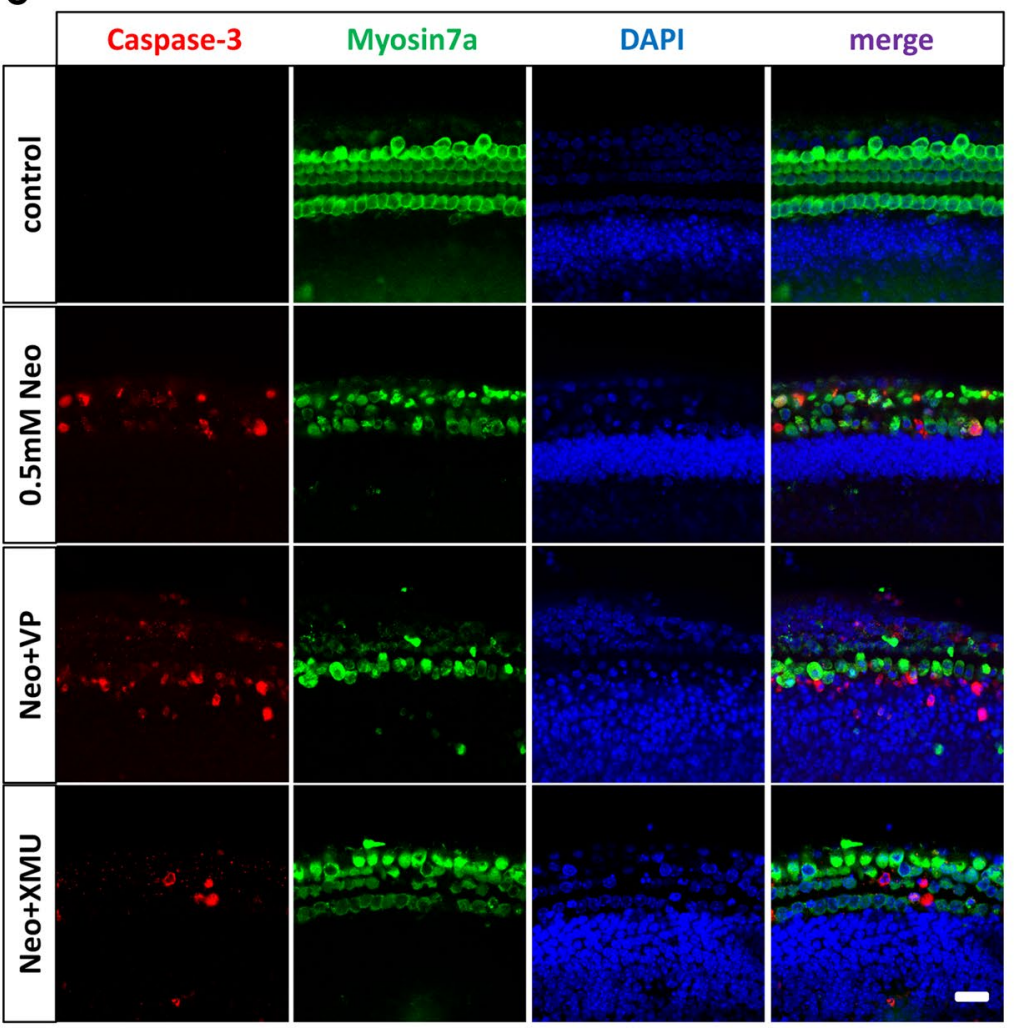

B

control

$0.5 \mathrm{mM} \mathrm{Neo}$

$0.5 \mathrm{mM} \mathrm{Neo}+2 \mu \mathrm{m}$ VP

$0.5 \mathrm{mM}$ Neo+1 $\mu \mathrm{m}$ XMU
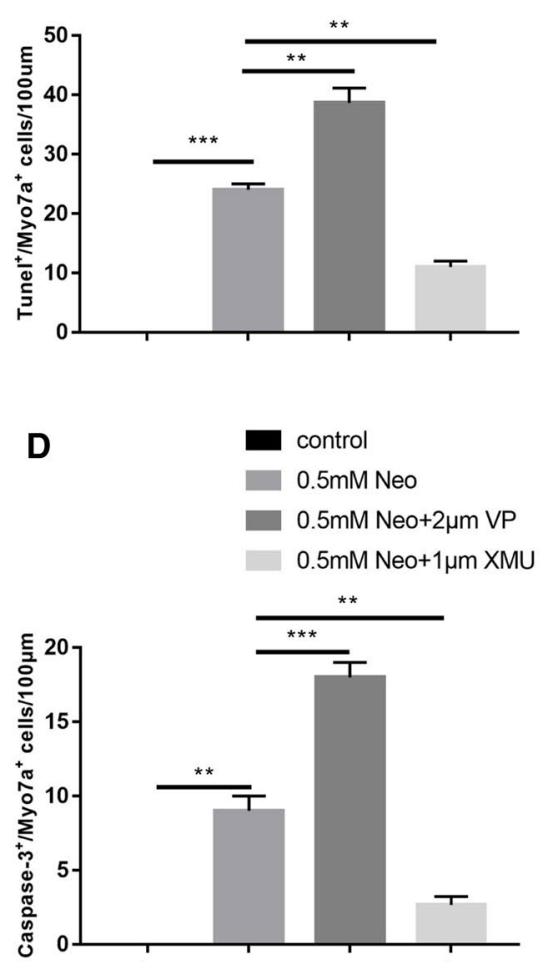

E

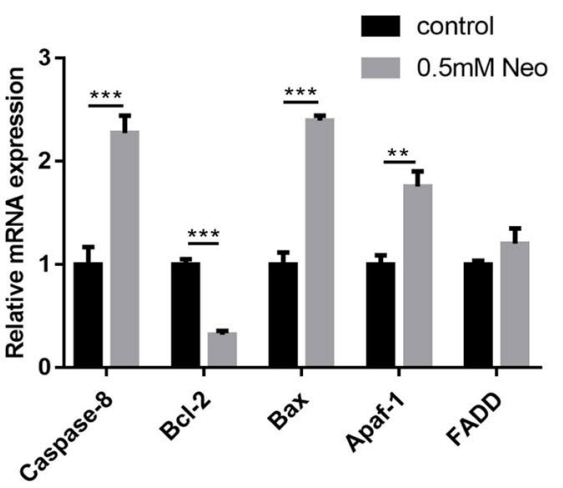

$\mathbf{F}$

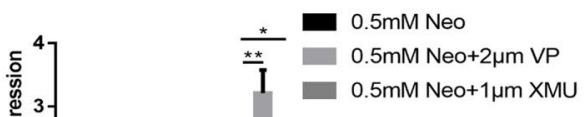

高

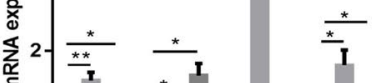

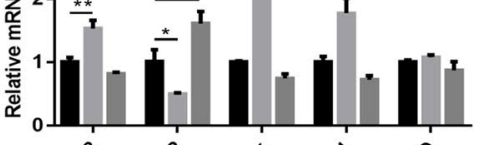

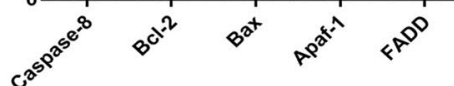


४Fig. 4 The Hippo/YAP signaling pathway regulates neomycininduced HC apoptosis in vitro. A Immunofluorescence staining with TUNEL and Myosin7a in the middle turn of the cochlear basilar membrane after different treatments. C Immunofluorescence staining with cleaved-Caspase 3 and Myosin7a in the middle turn of the cochlear basilar membrane after different treatments. B and D Quantification of the numbers of TUNEL $+/$ Myosin7a + double-positive cells and cleaved-Caspase $3+/$ Myosin $7 \mathrm{a}+$ double-positive cells in (A and $\mathbf{C}$ ). The numbers of TUNEL $+/$ Myosin7a + double-positive cells and cleaved-Caspase $3+/$ Myosin $7 \mathrm{a}+$ double-positive cells were significantly increased in the neomycin-treated group compared with the control group. Moreover, the numbers of apoptotic cells were significantly increased by VP treatment and decreased by XMU treatment. E The mRNA levels of apoptosis-related genes were analyzed by qPCR after neomycin treatment. F The mRNA levels of apoptosis-related genes were analyzed by qPCR in the VP-pretreatment group and XMU-pretreatment group after neomycin injury. $* p<0.05$, ${ }^{*} p<<0.01, * * * p<0.001, n=3$. Scale bars $=20 \mu \mathrm{m}$

the role of $\mathrm{C}-\mathrm{Abl}$ in neomycin-induced $\mathrm{HC}$ apoptosis, we first dissected and cultured the cochleae from P3 WT mice and then treated them with neomycin together with XMU or VP (Fig. 7A). Immunofluorescence staining and western blot results demonstrated that C-Abl expression was significantly increased after neomycin treatment, and significantly decreased after pretreatment with $\mathrm{XMU}$, indicating that YAP inhibits C-Abl activity (Figs. 6C, 7B). We also performed qPCR to explore the expression level of C-Abl signaling downstream genes in the cochlea after different treatments. The qPCR results demonstrated that the expression of the pro-apoptotic genes $C$ - $A b l$ and $p 73$ was significantly increased after neomycin treatment compared to the control group (Fig. 7C). Simultaneously, the expression of the pro-apoptotic genes $C-A b l$ and $p 73$ was significantly increased after pretreatment of cochleae with the YAP inhibitor VP compared to the neomycin-only group. In contrast, the expression of the pro-apoptotic genes $C-A b l$ and $p 73$ was significantly decreased when the cochleae were treated with the YAP agonist XMU (Fig. 7D). Thus, YAP/ p73-dependent apoptosis requires phosphorylation by C-Abl, which is in agreement with the findings of previous studies [53, 54]. In conclusion, neomycin-induced $\mathrm{HC}$ apoptosis was mediated by C-Abl, and YAP overexpression could inhibit C-Abl-mediated HC apoptosis in cochlear HCs after neomycin damage.

\section{Discussion}

The Hippo signaling pathway is a highly evolutionarily conserved signaling pathway that was first discovered in Drosophila, and it is the most recently discovered member of the known signaling pathway families involved in the control of tissue development and organ size [21, 22]. The Hippo signaling pathway controls organ size by regulating cell proliferation, apoptosis, and stem cell self-renewal [55-57]. The core components of the Hippo signaling pathway include serine/threonine kinase cascades, transcriptional coactivators, and transcription factors. YAP is a key transcriptional coactivator downstream of the Hippo signaling pathway. Due to the activation of upstream kinases such as MST1/2 and LATS1/2, YAP phosphorylation occurs on the serine residues of five consistent HXRXXS motifs in vitro and in vivo $[16,58,59]$. YAP phosphorylation is located at the S127 site and interacts with 14-3-3 to inhibit the transcriptional activity of YAP, and this causes YA to remain in the cytoplasm and participate in the regulation of cell apoptosis. In contrast, when upstream kinases are inactivated, unphosphorylated YAP can bind to downstream TEAD family members (such as TEAD1-4) to enter the nucleus and participate in the regulation of cell proliferation, differentiation, and cell survival [60]. Protein phosphatase-1 specifically dephosphorylates YAP at S127 and promotes the nuclear accumulation and transcriptional activity of YAP [61]. Enhancing YAP expression contributes to wound repair and tissue regeneration after inflammatory injury [62].

Hearing loss is a common clinical sensory disorder affecting 466 million people worldwide and occurs mainly as a result of HC damage. Ototoxic drugs, viral infections, genetic susceptibility, aging, and noise can all lead to irreversible sensorineural hearing loss. Ototoxic drugs, especially aminoglycosides, cause permanent hearing loss in approximately half a million people each year in the USA [63]. Aminoglycosides enter HCs through specific membrane channels and endocytosis, and accumulate to cytotoxic levels, ultimately resulting in HC death [64, 65]. Because $\mathrm{HCs}$ do not have regenerative capacity in mature mammals, protection against $\mathrm{HC}$ damage is critical to maintaining hearing function. An increasing number of studies have focused on the mechanisms and therapeutic approaches of HC loss caused by ototoxic drugs [66], and the Hippo/YAP signaling pathway has been shown to play an important regulatory role in the repair and regeneration of tissues such as the eyes, brain, kidney, heart, liver, and skin [30-35]. Unfortunately, there are few studies on the role of the Hippo/YAP signaling pathway in auditory organs. Recently, YAP has been reported to be required for $\mathrm{HC}$ proliferation and differentiation in the inner ear [67-69]. However, whether the Hippo/ YAP signaling pathway is required for $\mathrm{HC}$ survival has not been previously investigated.

To further explore the specific role and regulatory mechanism of the Hippo signaling pathway in HC damage protection in the mouse cochlea, we first verified the expression and localization of YAP in the mouse cochlea. We dissected cochleae from P3/P30 WT mice and cultured 
A

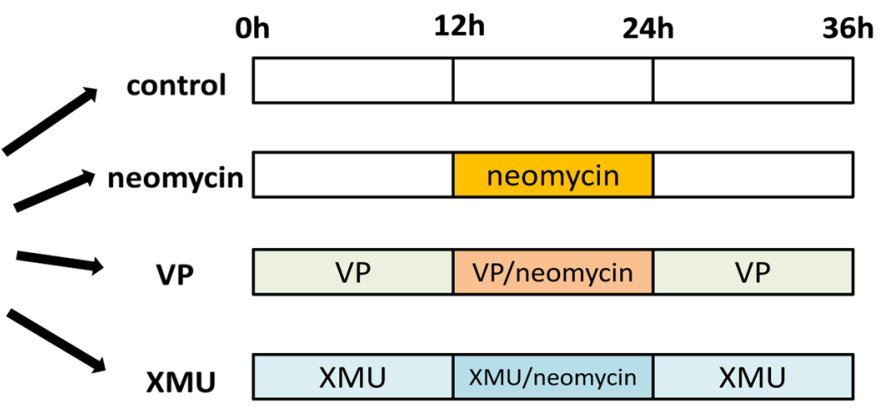

B
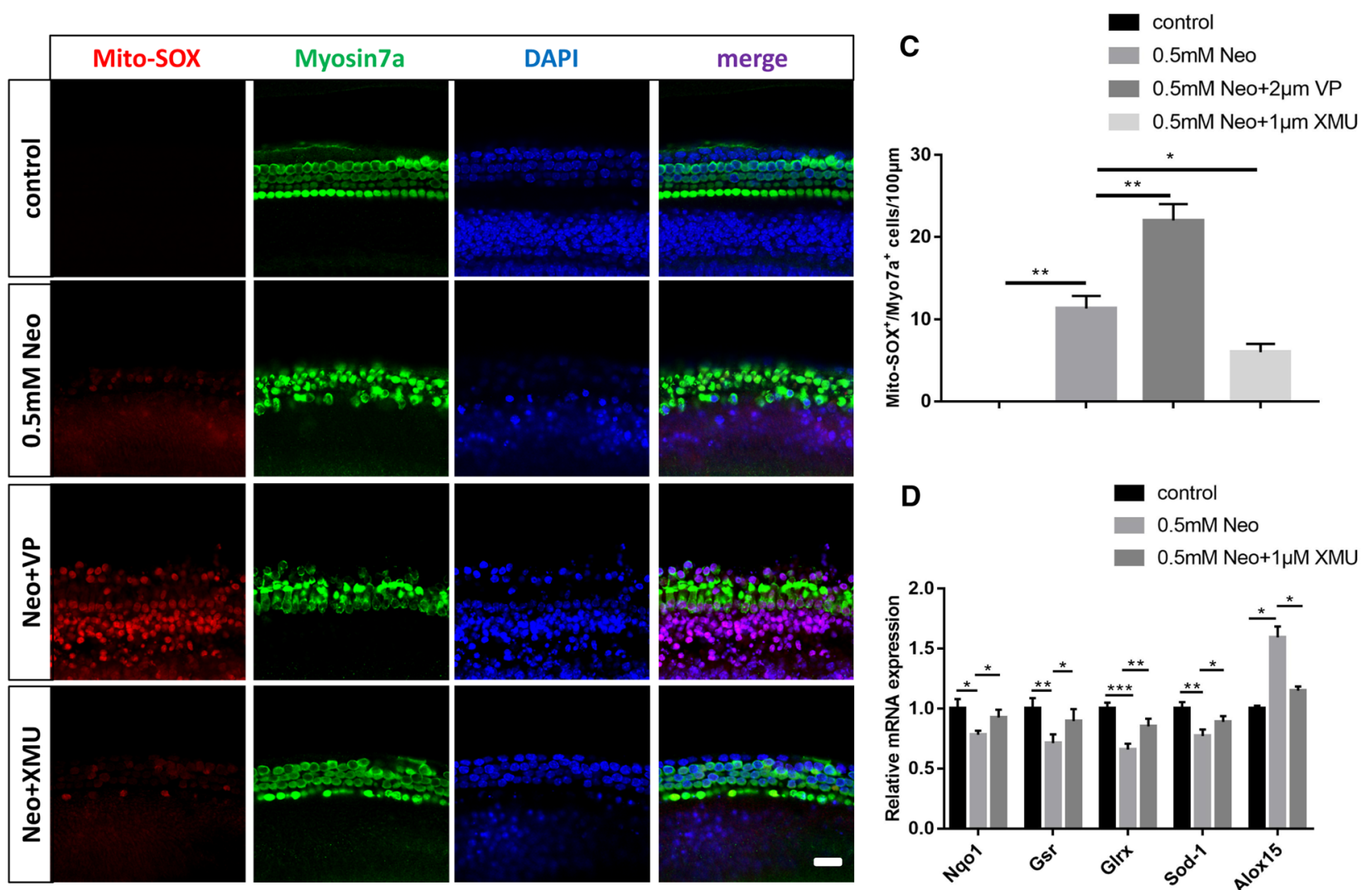

Fig. 5 The Hippo/YAP signaling pathway regulates the ROS levels in cochlear HCs after neomycin injury. A Schematic diagram of drug addition in tissue culture (divided into four groups). B Immunofluorescence staining with Mito-SOX and Myosin7a in the middle turn of the cochlear basilar membrane after different treatments. C Quantification of the numbers of Mito-SOX $+/$ Myosin7a + doublepositive cells in $(\mathbf{B})$. The numbers of Mito-SOX $+/$ Myosin $7 \mathrm{a}+$ dou-

them in vitro. The immunofluorescence results showed that YAP was expressed in the cytoplasm of cochlear HCs, which is consistent with the biological characteristics of YAP, in which, under normal conditions, YAP remains in the cytoplasm and is targeted for degradation [70]. We next used ble-positive cells were significantly increased in the neomycin-treated group compared with the control group. In addition, the neomycininduced oxidative stress was significantly increased by VP treatment and decreased by XMU treatment. D The mRNA levels of redoxrelated genes were analyzed by qPCR in the neomycin-only group and the XMU-pretreatment group after neomycin injury. ${ }^{*} p<0.05$, $* * p<0.01, * * * p<0.001, n=3$. Scale bars $=20 \mu \mathrm{m}$

the aminoglycoside drug neomycin to specifically damage HCs to construct an HC injury model. We found that the expression of YAP was decreased after neomycin injury. Hippo/YAP signaling pathway was activated by XMU in cochlear HCs after neomycin treatment, indicating that the 
Hippo/YAP signaling pathway is a protective physiological mechanism against neomycin injury.

We hypothesized that neomycin-induced HC loss could be prevented by regulating the expression level of YAP. To verify this hypothesis, we used the YAP agonist XMU and the YAP inhibitor VP to upregulate and downregulate the expression level of YAP, respectively. The pharmacokinetic characteristics of XMU could specifically inhibit the activity of MST1/2 in mice [71, 72], leading to activation of downstream YAP. VP has been used as a photosensitizer for photodynamic therapy, which has been approved by the Food and Drug Administration for treating age-related macular degeneration, pathological myopia, and neovascularization due to putative ocular histoplasmosis [73]. A recent highthroughput drug screening study reported that VP could inhibit the transcriptional activation of YAP-TEAD, leading to inhibition of cell proliferation and cell survival, and promotion of cell apoptosis [74].

In this study, we also found that the optimal concentration of XMU was $1 \mu \mathrm{M}$ and that the optimal concentration of VP was $2 \mu \mathrm{M}$. The appropriate concentration of XMU or VP was used to pretreat the dissected cochleae, which were then cultured with neomycin in vitro. The immunofluorescence results showed that $\mathrm{HC}$ loss was significantly increased after neomycin injury compared to the control group. XMU significantly reduced the neomycin-induced $\mathrm{HC}$ loss, and VP significantly increased the neomycin-induced HC loss, indicating that neomycin-induced $\mathrm{HC}$ loss was significantly decreased after upregulation of YAP and increased after downregulation of YAP. These results are consistent with those of previous studies in other tissues such as the retina, brain, liver, kidney, and heart [30-35], all of which indicated that YAP plays a vital role in $\mathrm{HC}$ damage protection.

Several pathways are involved in aminoglycoside-induced HC damage, including cell apoptosis and oxidative stress $[75,76]$. Previous studies have demonstrated that one of the most common causes of HC death is the production of ROS [77]. Oxidative stress inhibits the endogenous antioxidant systems of the cochlea, leading to excessive production of ROS, disrupting redox balance, triggering mitochondrial depolarization, and activating caspase-3-mediated apoptosis $[78,79]$. In our study, we used MitoSox to detect the levels of ROS. Our results showed that XMU significantly reduced the ROS levels in cochlear HCs after neomycin exposure compared to the neomycin-only group, indicating that the Hippo/YAP signaling pathway can regulate the ROS levels. We next used TUNEL and Caspase-3 to label the apoptotic cells. The immunofluorescence results showed that the numbers of Myosin7a +/TUNEL + and Myosin7a +/Caspase3 + double-positive cells in the XMU/ neomycin-treated group were significantly decreased compared to the neomycin-only group. In addition, the results of RT-qPCR demonstrated that the expression of the proapoptotic genes caspase-8, Bax, and Apafl was significantly decreased, while the expression of the anti-apoptotic gene $B c l-2$ was significantly increased after XMU treatment. This suggests that the Hippo/YAP signaling pathway can regulate caspase-mediated apoptosis in cochlear HCs after neomycin injury. Activation of YAP inhibits HC apoptosis, while inhibition of YAP promotes HC apoptosis. In conclusion, the Hippo/YAP signaling pathway protects against neomycin-induced HC damage by reducing the ROS levels; thus, decreasing caspase-mediated cell apoptosis. However, this is not the sole mechanism responsible for aminoglycosideinduced $\mathrm{HC}$ death.

YAP acts as a tumor suppressor by activating p73 (a member of the downstream 553 family) in response to DNA damage. YAP binds to the PPPY motif of p73 through its WW domain and acts as a transcriptional coactivator of p73; thus, inducing the transcription of pro-apoptotic genes such as Bax [80]. C-Abl (Abelson murine leukemia viral oncogene) is a non-receptor tyrosine kinase that is activated to phosphorylate p73 at the Tyr99; thus, supporting the p73-dependent induction of apoptosis. At the same time, activated C-Abl also phosphorylates YAP at the Tyr357, and tyrosine-phosphorylated YAP accumulates on the targeted apoptotic gene promoter, where it preferentially binds to p73. Therefore, C-Abl-mediated phosphorylation of YAP increases the binding affinity of YAP to $\mathrm{p} 73$ by inhibiting the transcriptional activity of YAP-TEAD; thus, activating the pro-apoptotic target protein and transforming the activity of YAP from anti-apoptotic to pro-apoptotic [51, 52, 81]. To further investigate the effect of the C-Abl/ p73 transduction pathway in neomycin-induced $\mathrm{HC}$ damage in the mouse cochlea, we first verified the expression and localization of $\mathrm{C}$-Abl. Immunofluorescence results showed that C-Abl was strongly expressed in the nucleus of HCs. In the following in vitro experiment, we observed the changes in major effector factors, such as C-Abl, YAP, TEAD, and p73, in different treatment groups at the RNA and protein levels. We found that the expression of C-Abl and p73 was significantly increased, while the expression of YAP and TEAD were decreased after neomycin injury. These findings indicate that neomycin exposure activates $\mathrm{C}$-Abl and phosphorylates downstream YAP and that the phosphorylation of YAP leads to interaction with p73 and subsequent cell apoptosis and loss of HCs. Furthermore, YAP overexpression inhibited the activation of C-Abl; thus, inhibiting C-Abl-mediated cell apoptosis and suggesting that $\mathrm{C}$-Abl-mediated $\mathrm{HC}$ apoptosis is regulated by the Hippo signaling pathway. 
A
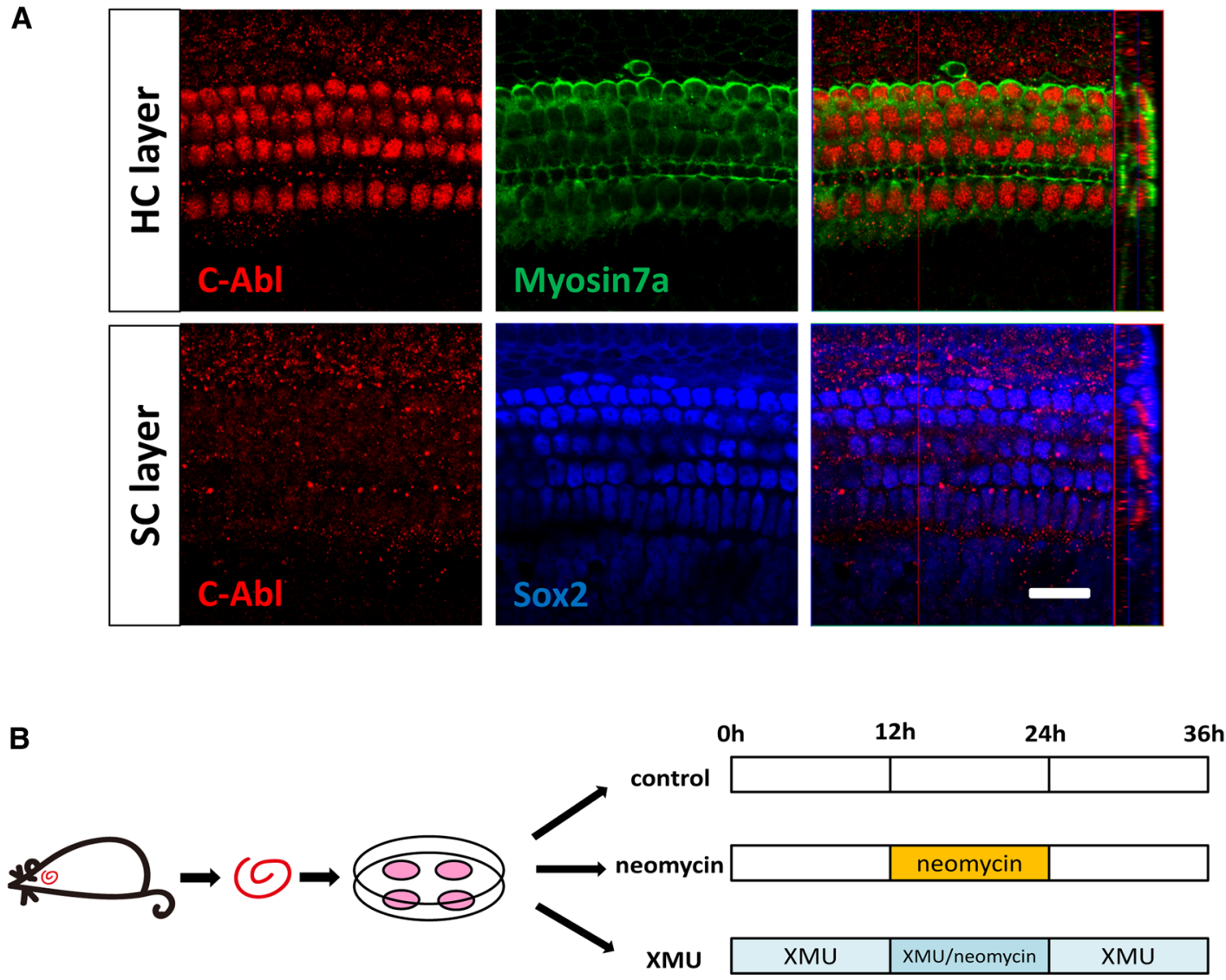

C
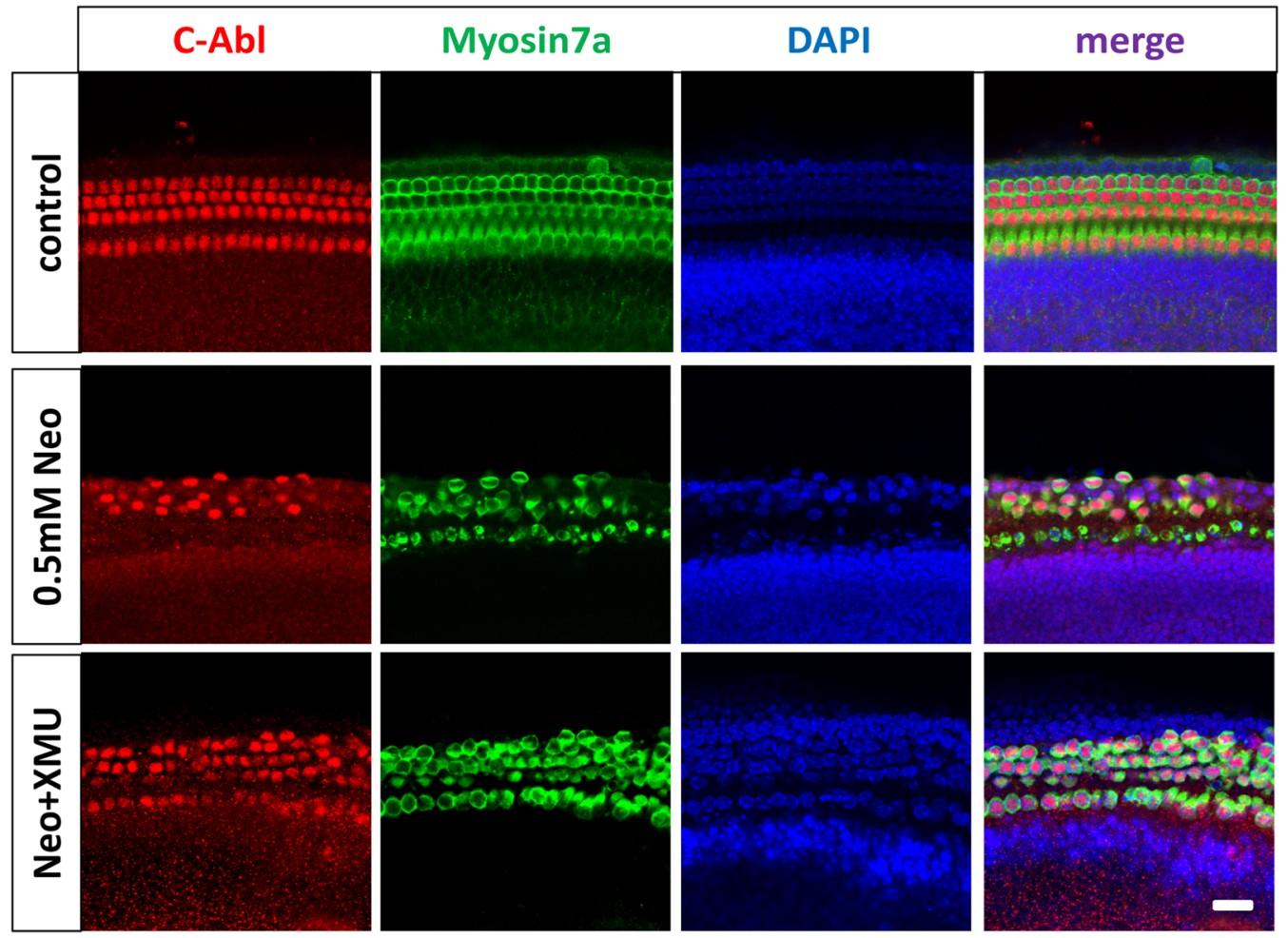
4Fig. 6 C-Abl expression is regulated by the Hippo/YAP signaling pathway in cochlear HCs after neomycin exposure. A The cochleae were dissected from P3 WT mice, and immunolabeled with Myosin7a (green), Sox2 (blue), and C-Abl (red). Immunofluorescence staining showed the expression and localization of $\mathrm{C}-\mathrm{Abl}$ in the P3 WT mouse cochlea under a $63 \times$ microscope, and C-Abl was expressed in the nuclei of cochlear HCs. B Schematic diagram of drug addition in tissue culture (divided into three groups). C Immunofluorescence staining with $\mathrm{C}-\mathrm{Abl}$ and Myosin $7 \mathrm{a}$ in the middle turn of the cochlear basilar membrane after different treatments. The expression of $\mathrm{C}$-Abl was significantly increased in the neomycintreated group and decreased in the XMU/neomycin-treated group. ${ }^{*} p<0.05, * * p<0.01$, *** $p<0.001, n=3$. Scale bars $=20 \mu \mathrm{m}$

\section{Conclusion}

In summary, we present the first investigation into the role of the Hippo/YAP signaling pathway in HC damage protection in the mouse cochlea. We showed that downregulated YAP expression increased neomycin-induced HC loss, and that YAP overexpression decreased neomycin-induced HC loss. We next demonstrated that the Hippo/YAP signaling pathway could regulate C-Abl-mediated $\mathrm{HC}$ apoptosis and ROS levels, which protects against neomycin-induced $\mathrm{HC}$ loss after neomycin exposure. Our results suggest that the
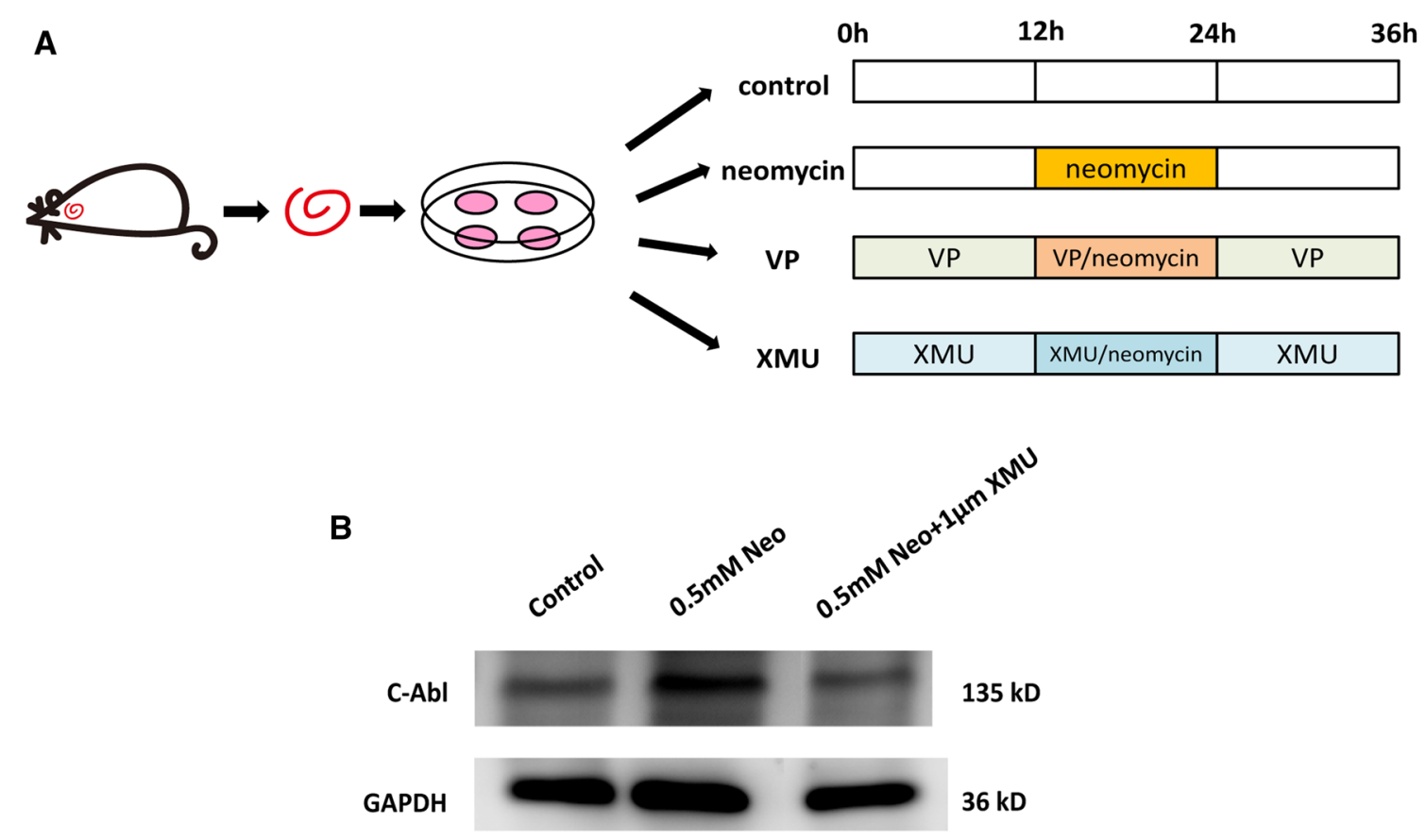

C

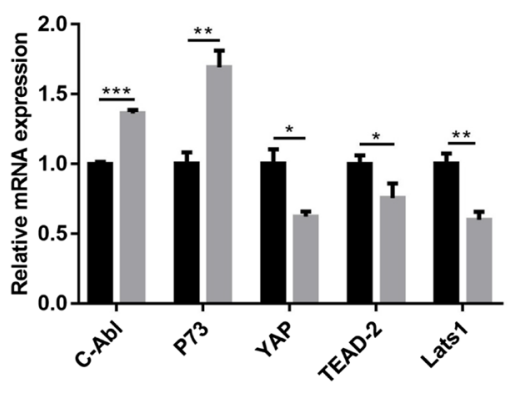

Fig. 7 YAP Overexpression inhibits C-Abl-mediated HC apoptosis in cochlear HCs after neomycin damage. A Schematic diagram of drug addition in tissue culture (divided into four groups). B The cochleae were dissected from P3 WT mice used for Western blot experiment. Western blot showed that the expression of C-Abl was significantly increased in the neomycin-treated group and decreased in the XMU/ without neomycin $0.5 \mathrm{mM}$ neomycin

D

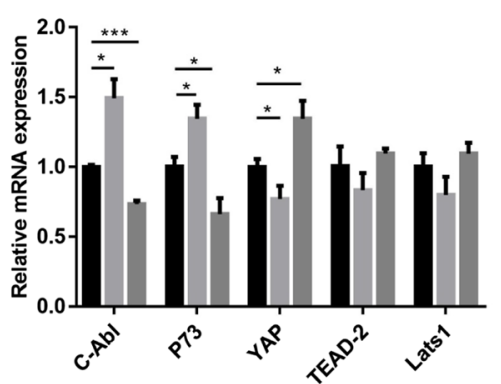

neomycin-treated group. C, D The mRNA levels of C-Abl signaling downstream genes were analyzed by qPCR in the different treatment groups. The qPCR results showed that XMU downregulated the expression of $\mathrm{C}-\mathrm{Abl}$ and $\mathrm{p} 73$ and that VP upregulated the expression of C-Abl and p73. ${ }^{*} p<0.05,{ }^{*} p<0.01, * * * p<0.001, n=3$. Scale bars $=20 \mu \mathrm{m}$ 
Hippo/YAP signaling pathway plays an essential role in HC damage protection and thus represents a novel therapeutic target for aminoglycoside-induced HC injury.

Acknowledgements This work was supported by grants from the National Key R\&D Program of China (No. 2021YFA1101300, 2020YFA0112503), the Strategic Priority Research Program of the Chinese Academy of Science (XDA16010303), the National Natural Science Foundation of China (Nos. 82030029, 81970884, $81900941,81900944,81970882,81870721,81771019$, $81700913)$, the Natural Science Foundation of Jiangsu Province (No. BE2019711, BK20190121), Science and Technology Department of Sichuan Province (No. 2021YFS0371), the Shenzhen Fundamental Research Program (JCYJ20190814093401920, JCYJ20210324125608022), Open Research Fund of State Key Laboratory of Genetic Engineering, Fudan University (No. SKLGE-2109), the Nanjing Medical Science and technique Development Foundation (QRX17051), Boehringer Ingelheim Pharma GmbH, and the Project of Invigorating Health Care Through Science, Technology and Education (ZDXKB2016015).

Author contributions M.W. and Y.D. conceived the study. B.G., C.Y., Y.Y., and R.C. supervised the experimental design. S.G., Z.Z., and C.C. acquired the data. M.W., Y.D., S.G., R.Q., and Y.Z. conducted the experiments. M.W., Y.D., and X.S. performed the statistical analysis. X.Q. and X.G. supervised the interpretation of the data. B.G., C.Y., Y.Y., and R.C. revised the manuscript and provided funding support. M.W. and Y.D. wrote the manuscript. All authors made substantial and direct intellectual contributions to this work and approved it for publication.

Availability of data and materials Not applicable.

Code availability Not applicable.

\section{Declarations}

Conflict of interest The authors declare that they have no conflict of interest.

Ethical approval Not applicable.

Consent to participate Not applicable.

Consent for publication All authors consent the publication of the manuscript in CMLS.

Open Access This article is licensed under a Creative Commons Attribution 4.0 International License, which permits use, sharing, adaptation, distribution and reproduction in any medium or format, as long as you give appropriate credit to the original author(s) and the source, provide a link to the Creative Commons licence, and indicate if changes were made. The images or other third party material in this article are included in the article's Creative Commons licence, unless indicated otherwise in a credit line to the material. If material is not included in the article's Creative Commons licence and your intended use is not permitted by statutory regulation or exceeds the permitted use, you will need to obtain permission directly from the copyright holder. To view a copy of this licence, visit http://creativecommons.org/licenses/by/4.0/.

\section{References}

1. Ma Y, Wise AK, Shepherd RK et al (2019) New molecular therapies for the treatment of hearing loss. Pharmacol Ther 200:190-209. https://doi.org/10.1016/j.pharmthera.2019.05.003

2. Petersen MB, Willems PJ (2006) Non-syndromic, autosomalrecessive deafness. Clin Genet 69:371-392. https://doi.org/10. 1111/j.1399-0004.2006.00613.x

3. Schacht J, Talaska AE, Rybak LP (2012) Cisplatin and aminoglycoside antibiotics: hearing loss and its prevention. Anat Rec 295:1837-1850. https://doi.org/10.1002/ar.22578

4. Prasad KN, Bondy SC (2020) Increased oxidative stress, inflammation, and glutamate: Potential preventive and therapeutic targets for hearing disorders. Mech Ageing Dev 185:111191. https://doi.org/10.1016/j.mad.2019.111191

5. Cunningham LL, Tucci DL (2017) Hearing loss in adults. N Engl J Med 377:2465-2473. https://doi.org/10.1056/NEJMr a1616601

6. Cianfrone G, Pentangelo D, Cianfrone F et al (2011) Pharmacological drugs inducing ototoxicity, vestibular symptoms and tinnitus: a reasoned and updated guide. Eur Rev Med Pharmacol Sci 15:601-636

7. Lanvers-Kaminsky C, Zehnhoff-Dinnesen AA, Parfitt R et al (2017) Drug-induced ototoxicity: Mechanisms, Pharmacogenetics, and protective strategies. Clin Pharmacol Ther 101:491500. https://doi.org/10.1002/cpt.603

8. Huth ME, Han KH, Sotoudeh K et al (2015) Designer aminoglycosides prevent cochlear hair cell loss and hearing loss. J Clin Invest 125:583-592. https://doi.org/10.1172/JCI77424

9. Justice RW, Zilian O, Woods DF et al (1995) The Drosophila tumor suppressor gene warts encodes a homolog of human myotonic dystrophy kinase and is required for the control of cell shape and proliferation. Genes Dev 9:534-546. https://doi.org/ 10.1101/gad.9.5.534

10. Tapon N, Harvey KF, Bell DW et al (2002) Salvador promotes both cell cycle exit and apoptosis in Drosophila and is mutated in human cancer cell lines. Cell 110:467-478. https://doi.org/ 10.1016/s0092-8674(02)00824-3

11. Harvey KF, Pfleger CM, Hariharan IK (2003) The Drosophila Mst ortholog, hippo, restricts growth and cell proliferation and promotes apoptosis. Cell 114:457-467. https://doi.org/10.1016/ s0092-8674(03)00557-9

12. Lai ZC, Wei X, Shimizu T et al (2005) Control of cell proliferation and apoptosis by mob as tumor suppressor, mats. Cell 120:675-685. https://doi.org/10.1016/j.cell.2004.12.036

13. Yu FX, Zhao B, Guan KL (2015) Hippo pathway in organ size control, tissue homeostasis, and cancer. Cell 163:811-828. https://doi.org/10.1016/j.cell.2015.10.044

14. Huang J, Wu S, Barrera J et al (2005) The Hippo signaling pathway coordinately regulates cell proliferation and apoptosis by inactivating Yorkie, the Drosophila Homolog of YAP. Cell 122:421-434. https://doi.org/10.1016/j.cell.2005.06.007

15. Zhao B, Li L, Lei Q et al (2010) The Hippo-YAP pathway in organ size control and tumorigenesis: an updated version. Genes Dev 24:862-874. https://doi.org/10.1101/gad.1909210

16. Zhao B, Wei X, Li W et al (2007) Inactivation of YAP oncoprotein by the Hippo pathway is involved in cell contact inhibition and tissue growth control. Genes Dev 21:2747-2761. https:// doi.org/10.1101/gad.1602907

17. Mo JS, Park HW, Guan KL (2014) The Hippo signaling pathway in stem cell biology and cancer. EMBO Rep 15:642-656. https://doi.org/10.15252/embr.201438638

18. Zhao B, Ye X, Yu J et al (2008) TEAD mediates YAP-dependent gene induction and growth control. Genes Dev 22:1962-1971. https://doi.org/10.1101/gad.1664408 
19. Edgar BA (2006) From cell structure to transcription: hippo forges a new path. Cell 124:267-273. https://doi.org/10.1016/j.cell.2006. 01.005

20. Harvey K, Tapon N (2007) The Salvador-Warts-Hippo pathway-an emerging tumour-suppressor network. Nat Rev Cancer 7:182-191. https://doi.org/10.1038/nrc2070

21. Pan D (2010) The hippo signaling pathway in development and cancer. Dev Cell 19:491-505. https://doi.org/10.1016/j.devcel. 2010.09.011

22. Halder G, Johnson RL (2011) Hippo signaling: growth control and beyond. Development 138:9-22. https://doi.org/10.1242/dev. 045500

23. Zheng Y, Pan D (2019) The hippo signaling pathway in development and disease. Dev Cell 50:264-282. https://doi.org/10.1016/j. devcel.2019.06.003

24. Taniguchi K, Wu LW, Grivennikov SI et al (2015) A gp130-SrcYAP module links inflammation to epithelial regeneration. Nature 519:57-62. https://doi.org/10.1038/nature14228

25. Park HW, Guan KL (2013) Regulation of the Hippo pathway and implications for anticancer drug development. Trends Pharmacol Sci 34:581-589. https://doi.org/10.1016/j.tips.2013.08. 006

26. Zhang W, Gao Y, Li P et al (2014) VGLL4 functions as a new tumor suppressor in lung cancer by negatively regulating the YAP-TEAD transcriptional complex. Cell Res 24:331-343. https://doi.org/10.1038/cr.2014.10

27. Xu K, Zhao H, Qiu X et al (2020) VGLL4 protects against oxidized-LDL-induced endothelial cell dysfunction and inflammation by activating hippo-YAP/TEAD1 signaling pathway. Mediators Inflamm 2020:8292173. https://doi.org/10.1155/ 2020/8292173

28. Gibault F, Corvaisier M, Bailly F et al (2016) Non-photoinduced biological properties of verteporfin. Curr Med Chem 23:11711184. https://doi.org/10.2174/0929867323666160316125048

29. Moya IM, Halder G (2019) Hippo-YAP/TAZ signalling in organ regeneration and regenerative medicine. Nat Rev Mol Cell Biol 20:211-226. https://doi.org/10.1038/s41580-018-0086-y

30. Kim JY, Park R, Lee JH et al (2016) Yap is essential for retinal progenitor cell cycle progression and RPE cell fate acquisition in the developing mouse eye. Dev Biol 419:336-347. https://doi.org/ 10.1016/j.ydbio.2016.09.001

31. Bai H, Zhang N, Xu Y et al (2012) Yes-associated protein regulates the hepatic response after bile duct ligation. Hepatology 56:1097-1107. https://doi.org/10.1002/hep.25769

32. Park R, Moon UY, Park JY et al (2016) Yap is required for ependymal integrity and is suppressed in LPA-induced hydrocephalus. Nat Commun 7:10329. https://doi.org/10.1038/ncomms10329

33. Lee MJ, Byun MR, Furutani-Seiki M et al (2014) YAP and TAZ regulate skin wound healing. J Invest Dermatol 134:518-525. https://doi.org/10.1038/jid.2013.339

34. Matsuda T, Zhai P, Sciarretta $S$ et al (2016) NF2 activates hippo signaling and promotes ischemia/reperfusion injury in the heart. Circ Res 119:596-606. https://doi.org/10.1161/CIRCRESAHA. 116.308586

35. Campbell KN, Wong JS, Gupta R et al (2013) Yes-associated protein (YAP) promotes cell survival by inhibiting proapoptotic dendrin signaling. J Biol Chem 288:17057-17062. https://doi.org/ 10.1074/jbc.C113.457390

36. Chen Y, Yu H, Zhang Y et al (2013) Cotransfection of Pax2 and Math1 promote in situ cochlear hair cell regeneration after neomycin insult. Sci Rep 3:2996. https://doi.org/10.1038/srep02996

37. Parker M, Brugeaud A, Edge AS (2010) Primary culture and plasmid electroporation of the murine organ of Corti. J Vis Exp. https://doi.org/10.3791/1685
38. Matsui JI, Ogilvie JM, Warchol ME (2002) Inhibition of caspases prevents ototoxic and ongoing hair cell death. J Neurosci 22:1218-1227

39. He Y, Yu H, Cai C et al (2015) Inhibition of H3K4me2 demethylation protects auditory hair cells from neomycin-induced apoptosis. Mol Neurobiol 52:196-205. https://doi.org/10.1007/ s12035-014-8841-3

40. Sun S, Sun M, Zhang Y et al (2014) In vivo overexpression of $\mathrm{X}$-linked inhibitor of apoptosis protein protects against neomycininduced hair cell loss in the apical turn of the cochlea during the ototoxic-sensitive period. Front Cell Neurosci 8:248. https://doi. org/10.3389/fncel.2014.00248

41. Sun $\mathrm{S}, \mathrm{Yu} \mathrm{H}, \mathrm{Yu} \mathrm{H}$ et al (2015) Inhibition of the activation and recruitment of microglia-like cells protects against neomycininduced ototoxicity. Mol Neurobiol 51:252-267. https://doi.org/ 10.1007/s12035-014-8712-y

42. Choung YH, Taura A, Pak K et al (2009) Generation of highlyreactive oxygen species is closely related to hair cell damage in rat organ of Corti treated with gentamicin. Neuroscience 161:214 226. https://doi.org/10.1016/j.neuroscience.2009.02.085

43. Balaban RS, Nemoto S, Finkel T (2005) Mitochondria, oxidants, and aging. Cell 120:483-495. https://doi.org/10.1016/j.cell.2005. 02.001

44. Huang T, Cheng AG, Stupak H et al (2000) Oxidative stressinduced apoptosis of cochlear sensory cells: otoprotective strategies. Int J Dev Neurosci 18:259-270. https://doi.org/10.1016/ s0736-5748(99)00094-5

45. Kalyanaraman B, Hardy M, Podsiadly R et al (2017) Recent developments in detection of superoxide radical anion and hydrogen peroxide: opportunities, challenges, and implications in redox signaling. Arch Biochem Biophys 617:38-47. https://doi.org/10. 1016/j.abb.2016.08.021

46. Liu J, Cao L, Chen J et al (2009) Bmil regulates mitochondrial function and the DNA damage response pathway. Nature 459:387-392. https://doi.org/10.1038/nature08040

47. Du H, Guo L, Fang F et al (2008) Cyclophilin D deficiency attenuates mitochondrial and neuronal perturbation and ameliorates learning and memory in Alzheimer's disease. Nat Med 14:10971105. https://doi.org/10.1038/nm.1868

48. Yousefi S, Mihalache C, Kozlowski E et al (2009) Viable neutrophils release mitochondrial DNA to form neutrophil extracellular traps. Cell Death Differ 16:1438-1444. https://doi.org/10.1038/ cdd.2009.96

49. Reuven N, Adler J, Meltser V et al (2013) The Hippo pathway kinase Lats2 prevents DNA damage-induced apoptosis through inhibition of the tyrosine kinase c-Abl. Cell Death Differ 20:1330 1340. https://doi.org/10.1038/cdd.2013.83

50. He M, Zhou Z, Shah AA et al (2016) New insights into posttranslational modifications of Hippo pathway in carcinogenesis and therapeutics. Cell Div 11:4. https://doi.org/10.1186/ s13008-016-0013-6

51. Keshet R, Adler J, Ricardo Lax I et al (2015) c-Abl antagonizes the YAP oncogenic function. Cell Death Differ 22:935-945. https://doi.org/10.1038/cdd.2014.182

52. Levy D, Adamovich Y, Reuven N et al (2008) Yap1 phosphorylation by c-Abl is a critical step in selective activation of proapoptotic genes in response to DNA damage. Mol Cell 29:350-361. https://doi.org/10.1016/j.molcel.2007.12.022

53. Gong JG, Costanzo A, Yang HQ et al (1999) The tyrosine kinase c-Abl regulates p73 in apoptotic response to cisplatin-induced DNA damage. Nature 399:806-809. https://doi.org/10.1038/ 21690 
54. Yuan ZM, Shioya H, Ishiko T et al (1999) p73 is regulated by tyrosine kinase c-Abl in the apoptotic response to DNA damage. Nature 399:814-817. https://doi.org/10.1038/21704

55. Zhao B, Tumaneng K, Guan KL (2011) The Hippo pathway in organ size control, tissue regeneration and stem cell self-renewal. Nat Cell Biol 13:877-883. https://doi.org/10.1038/ncb2303

56. Chen X, Li Y, Luo J et al (2020) Molecular mechanism of hippoYAP1/TAZ pathway in heart development, disease, and regeneration. Front Physiol 11:389. https://doi.org/10.3389/fphys.2020. 00389

57. Wang J, Liu S, Heallen T et al (2018) The Hippo pathway in the heart: pivotal roles in development, disease, and regeneration. Nat Rev Cardiol 15:672-684. https://doi.org/10.1038/ s41569-018-0063-3

58. Song H, Mak KK, Topol L et al (2010) Mammalian Mst1 and Mst2 kinases play essential roles in organ size control and tumor suppression. Proc Natl Acad Sci U S A 107:1431-1436. https:// doi.org/10.1073/pnas.0911409107

59. Dong J, Feldmann G, Huang J et al (2007) Elucidation of a universal size-control mechanism in Drosophila and mammals. Cell 130:1120-1133. https://doi.org/10.1016/j.cell.2007.07.019

60. Zhou Q, Li L, Zhao B et al (2015) The hippo pathway in heart development, regeneration, and diseases. Circ Res 116:14311447. https://doi.org/10.1161/CIRCRESAHA.116.303311

61. Wang P, Bai Y, Song B et al (2011) PP1A-mediated dephosphorylation positively regulates YAP2 activity. PLoS ONE 6:e24288. https://doi.org/10.1371/journal.pone.0024288

62. Yui S, Azzolin L, Maimets M et al (2018) YAP/TAZ-dependent reprogramming of colonic epithelium links ECM remodeling to tissue regeneration. Cell Stem Cell 22(35-49):e37. https://doi.org/ 10.1016/j.stem.2017.11.001

63. Francis SP, Kramarenko II, Brandon CS et al (2011) Celastrol inhibits aminoglycoside-induced ototoxicity via heat shock protein 32. Cell Death Dis 2:e195. https://doi.org/10.1038/cddis.2011.76

64. Hailey DW, Esterberg R, Linbo TH et al (2017) Fluorescent aminoglycosides reveal intracellular trafficking routes in mechanosensory hair cells. J Clin Invest 127:472-486. https://doi.org/10.1172/ JCI85052

65. Huth ME, Ricci AJ, Cheng AG (2011) Mechanisms of aminoglycoside ototoxicity and targets of hair cell protection. Int J Otolaryngol 2011:937861. https://doi.org/10.1155/2011/937861

66. Guo J, Chai R, Li H et al (2019) Protection of hair cells from ototoxic drug-induced hearing loss. Adv Exp Med Biol 1130:17-36. https://doi.org/10.1007/978-981-13-6123-4_2

67. Borse V, Barton M, Arndt $\mathrm{H}$ et al (2021) Dynamic patterns of YAP1 expression and cellular localization in the developing and injured utricle. Sci Rep 11:2140. https://doi.org/10.1038/ s41598-020-77775-8

68. Rudolf MA, Andreeva A, Kozlowski MM et al (2020) YAP mediates hair cell regeneration in balance organs of chickens, but LATS kinases suppress its activity in Mice. J Neurosci 40:3915-3932. https://doi.org/10.1523/JNEUROSCI.0306-20.2020
69. Gnedeva K, Wang X, McGovern MM et al (2020) Organ of Corti size is governed by Yap/Tead-mediated progenitor self-renewal. Proc Natl Acad Sci U S A 117:13552-13561. https://doi.org/10. 1073/pnas.2000175117

70. Panciera T, Azzolin L, Cordenonsi M et al (2017) Mechanobiology of YAP and TAZ in physiology and disease. Nat Rev Mol Cell Biol 18:758-770. https://doi.org/10.1038/nrm.2017.87

71. Fan F, He Z, Kong LL et al (2016) Pharmacological targeting of kinases MST1 and MST2 augments tissue repair and regeneration. Sci Transl Med 8:352ra108. https://doi.org/10.1126/scitranslmed. aaf 2304

72. Triastuti E, Nugroho AB, Zi M et al (2019) Pharmacological inhibition of Hippo pathway, with the novel kinase inhibitor XMUMP-1, protects the heart against adverse effects during pressure overload. Br J Pharmacol 176:3956-3971. https://doi.org/10.1111/ bph. 14795

73. Newman DK (2016) Photodynamic therapy: current role in the treatment of chorioretinal conditions. Eye (Lond) 30:202-210. https://doi.org/10.1038/eye.2015.251

74. Liu-Chittenden Y, Huang B, Shim JS et al (2012) Genetic and pharmacological disruption of the TEAD-YAP complex suppresses the oncogenic activity of YAP. Genes Dev 26:1300-1305. https://doi.org/10.1101/gad.192856.112

75. Dinh CT, Goncalves S, Bas E et al (2015) Molecular regulation of auditory hair cell death and approaches to protect sensory receptor cells and/or stimulate repair following acoustic trauma. Front Cell Neurosci 9:96. https://doi.org/10.3389/fncel.2015.00096

76. Karasawa T, Steyger PS (2011) Intracellular mechanisms of aminoglycoside-induced cytotoxicity. Integr Biol 3:879-886. https:// doi.org/10.1039/c1ib00034a

77. Peng TI, Jou MJ (2010) Oxidative stress caused by mitochondrial calcium overload. Ann N Y Acad Sci 1201:183-188. https://doi. org/10.1111/j.1749-6632.2010.05634.x

78. Wong AC, Ryan AF (2015) Mechanisms of sensorineural cell damage, death and survival in the cochlea. Front Aging Neurosci 7:58. https://doi.org/10.3389/fnagi.2015.00058

79. Xie J, Talaska AE, Schacht J (2011) New developments in aminoglycoside therapy and ototoxicity. Hear Res 281:28-37. https:// doi.org/10.1016/j.heares.2011.05.008

80. Strano S, Munarriz E, Rossi M et al (2001) Physical interaction with Yes-associated protein enhances p73 transcriptional activity. J Biol Chem 276:15164-15173. https://doi.org/10.1074/jbc. M010484200

81. Levy D, Reuven N, Shaul Y (2008) A regulatory circuit controlling Itch-mediated p73 degradation by Runx. J Biol Chem 283:27462-27468. https://doi.org/10.1074/jbc.M803941200

Publisher's Note Springer Nature remains neutral with regard to jurisdictional claims in published maps and institutional affiliations. 


\section{Authors and Affiliations}

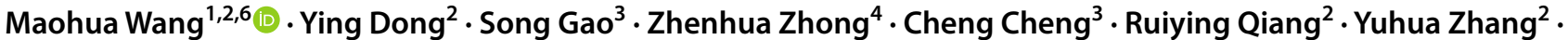

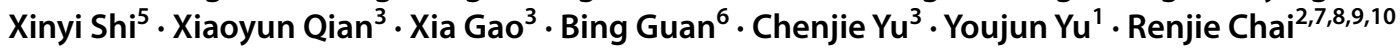

1 Department of Otolaryngology, Head and Neck Surgery, The First People's Hospital of Foshan, Affiliated Foshan Hospital of Sun Yat-Sen University, Hearing and Balance Medical Engineering Technology Center of Guangdong, Foshan 528000, China

2 State Key Laboratory of Bioelectronics, Department of Otolaryngology Head and Neck Surgery, Zhongda Hospital, School of Life Sciences and Technology, Jiangsu Province High-Tech Key Laboratory for Bio-Medical Research, Southeast University, Nanjing 210096, China

3 Department of Otolaryngology, Head and Neck Surgery, Affiliated Drum Tower Hospital of Nanjing University Medical School, Jiangsu Provincial Key Medical Discipline (Laboratory), Nanjing 210008, China

4 Department of Otolaryngology, Head and Neck Surgery, The Affiliated Hospital of Yangzhou University, Yangzhou University, Yangzhou 225000, China
5 Department of Otolaryngology, Head and Neck Surgery, Affiliated Jiangning Hospital of Nanjing Medical University, Nanjing 211100, China

6 Department of Otolaryngology, Head and Neck Surgery, Clinical Medical College, Yangzhou University, Yangzhou 225001, China

7 Department of Otolaryngology Head and Neck Surgery, Sichuan Provincial People's Hospital, University of Electronic Science and Technology of China, Chengdu 610072, China

8 Co-Innovation Center of Neuroregeneration, Nantong University, Nantong 226001, China

9 Institute for Stem Cell and Regeneration, Chinese Academy of Science, Beijing, China

10 Beijing Key Laboratory of Neural Regeneration and Repair, Capital Medical University, Beijing 100069, China 